\title{
Graphene-based nanomaterials in biosystems
}

\author{
$\mathrm{Na} \mathrm{Lu}{ }^{1}$, Liqian Wang ${ }^{2}$, Min Lv², Zisheng $\operatorname{Tang}^{3,4,5}(\varangle)$, and Chunhai $\operatorname{Fan}^{2,6}(\bowtie)$ \\ ${ }^{1}$ School of Materials Engineering, Shanghai University of Engineering Science, Shanghai 201620, China \\ ${ }^{2}$ Division of Physical Biology and Bioimaging Center, Shanghai Synchrotron Radiation Facility, CAS Key Laboratory of Interfacial Physics and \\ Technology, Shanghai Institute of Applied Physics, Chinese Academy of Sciences, Shanghai 201800, China \\ ${ }^{3}$ Department of Endodontics, Shanghai Ninth People's Hospital, College of Stomatology, Shanghai Jiao Tong University School of Medicine, Shanghai \\ 200011, China \\ ${ }^{4}$ National Clinical Research Center of Oral Diseases, Shanghai 200011, China \\ ${ }^{5}$ Shanghai Key Laboratory of Stomatology \& Shanghai Research Institute of Stomatology, Shanghai 200011, China \\ ${ }^{6}$ School of Chemistry and Chemical Engineering, and Institute of Molecular Medicine, Renji Hospital, School of Medicine, Shanghai Jiao Tong \\ University, Shanghai 200240, China
}

(c) Tsinghua University Press and Springer-Verlag GmbH Germany, part of Springer Nature 2018

Received: 12 June 2018 / Revised: 12 September 2018 / Accepted: 14 September 2018

\begin{abstract}
Graphene-based nanomaterials have emerged as a novel type of materials with exceptional physicochemical properties and numerous applications in various areas. In this review, we summarize recent advances in studying interactions between graphene and biosystems. We first provide a brief introduction on graphene and its derivatives, and then discuss on the toxicology and biocompatibility of graphene, including the extracellular interactions between graphene and biomacromolecules, cellular studies of graphene, and in vivo toxicological effects. Next, we focus on various graphene-based practical applications in antibacterial materials, wound addressing, drug delivery, and water purification. We finally present perspectives on challenges and future developments in these exciting fields.
\end{abstract}

\section{KEYWORDS}

graphene-based nanomaterials, toxicology and biocompatibility, biomacromolecules, cells, living entities, applications

\section{Introduction}

Graphene is the fundamental building element of many carbon allotropes including graphite, charcoals, carbon nanotubes (CNTs) and bucky balls. Since its discovery in 2004 [1], graphene has rapidly emerged as a family of highly promising nanomaterials with unique electronic, optical and catalytic properties that can be exploited for numerous applications in energy, environment, and biomedicine. In particular, the coupling of graphene with biology brings exciting research focuses. Due to their large surface areas, superior physical and chemical properties, graphene-based nanomaterials has emerged as an attractive candidate to act as nanoscale building blocks for biological researches. The strong van der Waals force between graphene nanosheets facilitate incorporating molecules or nanomaterials (e.g., polymer or nanoparticles) to form multifunctional nanocomposites, which is effective to improve their properties and enhance their performance toward biosystems. In addition, based on their inherent antibacterial properties, graphene can be used as a new effective and green antibacterial agent with a severe cell inactivation toward bacteria. Also importantly, graphenebased nanomaterial is renewable and easy to fabricate with low cost and fast preparation techniques, compared to traditional metals and metal oxides. The research ranges from fundamental studies on the interactions between graphene and biomolecules to various graphenebased practical applications including antibacterial materials, cell culture substrates, biosensors and drug delivery carriers. While several excellent reviews have appeared with the focus on some of these topics [2-5], there has not been a comprehensive review that summarizes the research progress in graphene-based nanomaterials in biosystems.

In this review, we summarize recent advances in the biocompatibility, toxicology and applications of graphene-based nanomaterials in biosystems (Fig. 1). We first briefly describe the structures, properties

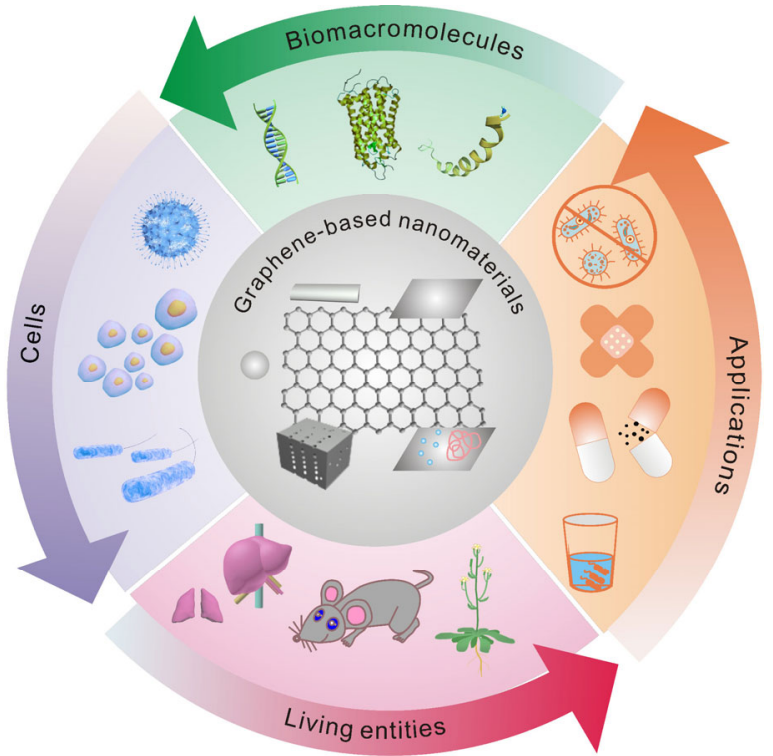

Figure 1 Overview of the biocompatibility, toxicology and applications of graphene-based nanomaterials in biosystems. 
and synthesis of graphene, graphene derivatives, and graphene-based composites. Sequentially, we focus on the recent advances in the toxicology and biocompatibility of graphene in biosystems, including the extracellular interactions between graphene and biomacromolecules (e.g., nucleic acids, proteins, and peptides), the way of graphene influences on the biological responses of viruses and cellular metabolism, how graphene enters cells, in vivo toxicological effects of graphene on animals and plants, and toxicological concerns regarding its potential applications. Next, we summarize the recent achievements and advancements in the antibacterial activities of graphene, its derivatives and graphene-based nanocomposites, the mechanisms of graphene-mediated antibacterial properties, and other applications in wound addressing, drug delivery, and water purification. Finally, we suggest some significant prospects, further developments and opportunities in this emerging and promising field.

\section{Brief descriptions of graphene-based nanomaterials}

\subsection{Graphene and graphene derivatives}

Graphene, a two-dimensional layer of $\mathrm{sp}^{2}$-hybridized single-atomscale carbon, has caught appreciable attention due to its extraordinary intrinsic strength, high surface area, fast electron mobility, high thermal conductivity, and strong Young's modulus. Each carbon atom in the lattice has the $\mathrm{s}, \mathrm{p}_{x}$, and $\mathrm{p}_{y}$ atomic orbitals that hybridize to form covalent $\mathrm{sp}^{2}$ bonds, which gives rise to $\mathrm{C}-\mathrm{C}-\mathrm{C}$ bond angles of $120^{\circ}$ and the chicken-wire-like layers. In the hexagonal ring, three valence electrons on each carbon in the lattice form the $\sigma$ bond, which makes carbon atoms bonded together. A $\pi$ orbital known as the valence band, is formed by the remaining $\mathrm{p}_{z}$ orbital on each carbon atom overlaps with its neighbors. The $\pi$ orbital is also called the conduction band that contributes to delocalized electrons. Graphene-based nanomaterials contain not only "pristine" graphene, but also chemical modified graphene as graphene derivatives that have been treated by chemical modifications [6] for covalent bonding with pendent groups, such as graphene oxide (GO) and reduced graphene oxide (rGO). GO contains epoxy and carbon radicals in the basal planes, while its edges are decorated with carboxyl and hydroxyl groups. The amount of oxygen groups depends significantly on the preparation approaches, including direct oxidation of graphite and subsequent exfoliation of carbon sheets that are deeply oxidized. The intrinsic properties of graphene make that GO can partly be reduced going toward graphene-like sheets, which is generally referred as rGO. Chemical doping is another effective approach to synthesize doped-graphene, which is used to tailor the nature of graphene. In doped graphene, other elements, such as nitrogen atoms, boron atoms, and sulfur atoms, are introduced into the hexagonal lattice of graphene and replace the carbon atoms. Usually, the doped-atoms are under $10 \%$ of the whole system. Except modifying the natural properties of graphene, the morphologies can also be shaped, such as two-dimensional (2D) graphene nanosheets (GNSs) [1], zero-dimensional (0D) graphene quantum dots (GQDs) [7], and one-dimensional (1D) graphene nanoribbons (GNRs) [8].

Up to now, the synthesis procedures of graphene and its derivatives can be classified into two main categories: physical and chemical approaches. Physical approaches utilize exfoliation of a graphene layer from bulk graphite by disrupting the van der Waals forces between the stacked sheets, including mechanical exfoliation, and direct liquid phase exfoliation. Chemical approaches build up various graphene-based nanomaterials through chemical reaction, including epitaxial growth, chemical vapor deposition (CVD), chemical oxidation, chemical reduction, and so on. The structures of graphene, its derivatives, and graphene-based composites as well as their related prepared approaches are shown in Fig. 2.

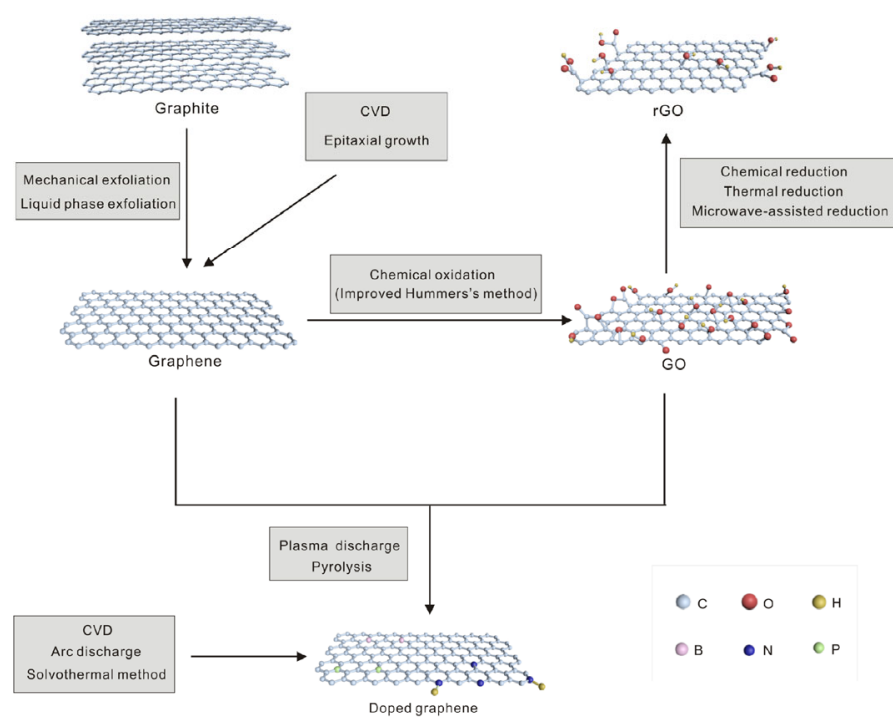

Figure 2 Graphene-based nanomaterials and their related preparation techniques.

\subsection{Physically grown graphene}

In graphite, the interactions between adjacent graphene layers formed via van der Waals forces are extremely weak [9]. This causes the delamination of bulk graphite into individual graphene sheets under mechanical forces. Geim and his co-workers first mechanically exfoliated graphene by using adhesive tapes in 2004 [1], which has been credited with the explosive growth of interest in graphene in recent years. This simple approach can keep the structural integrity of graphene; however, it has low efficiency, and the size and orientation of available graphene sheets are generally uncontrollable with the lateral size up to tens of microns.

Liquid-phase exfoliation is another typical physical synthesis approach, which is prepared by sonicating graphite in the presence of certain solvents. This direct method is based on one-step physical process by using liquids as exfoliation media, such as organic solvents, ionic liquids, and surfactant solutions. Coleman et al. [10] developed graphene dispersions in the forms of mono- and multilayered graphene sheets, which are free from oxides and defects. The yield of monolayer graphene in $\mathrm{N}$-methyl pyrrolidone (NMP) dispersions was $28 \%$, corresponding to an overall yield of approximately $1 \mathrm{wt} . \%$. However, the low concentration of obtained graphene, which was below $0.01 \mathrm{mg} \cdot \mathrm{mL}^{-1}$, made it difficult to concentrate and isolate from the viscous solvents.

\subsection{Chemically produced graphene}

CVD technique has been widely used to produce large-scale and high-quality graphene films with low/no oxygen content and defect-free hexagonal lattice. Hong, Choi and co-workers [11] used CVD technique to grow and transfer few-layer graphene films on a large scale on a $300 \mathrm{~nm}$-thick nickel layer on a $\mathrm{SiO}_{2} / \mathrm{Si}$ substrate. Besides nickel, copper $(\mathrm{Cu})$ is another ideal metal in CVD synthesis. Bea et al. [12] developed the roll-to-roll production of monolayer 30-inch graphene films through CVD technique onto flexible and large copper substrates. The films presented low resistances of $\sim 125 \Omega \cdot \mathrm{m}^{-1}$ and $97.4 \%$ optical transmittance, which was superior to that grown on indium tin oxides. Recently, Chen et al. [13] found the lattice constant of monolayer graphene as-grown on $\mathrm{Cu}$ via $\mathrm{CVD}$ was expanded to around $7.5 \%$ of its relaxed value using electronic and lattice structure analysis.

Epitaxial growth of graphene film formed by thermal decomposition and vacuum graphitization on insulating carbon-containing or metal substrate, has also been reported [14-16]. After Si atoms was evaporated from the substrate, uniform ordered graphene films in 
the size of few microns was produced on silicon carbide $(\mathrm{SiC})$ surface through vacuum high-temperature annealing and carbon segregation. This technique could obtain high-quality graphene films. Unfortunately, it requires special conditions of high temperature and vacuum environment, and expensive costs for only small-size graphene film.

The most common way to prepare graphene for biological researches is chemical reduction of GO to $\mathrm{rGO}$, involving deep oxidation of graphite, followed exfoliation of GO by sonication irritation, and reduction by chemical or thermal methods. Hummer's method [17] is used as the most common approach for producing GO. Currently, there are several improved techniques to Hummer oxidation by using different oxidizing agents/acids or under different reaction conditions. Ruoff et al. [18] prepared a free-standing GO paper by flow-directed assembly method, possessing higher tensile modulus and fracture strength than many other paper-like materials. Marcano et al. [19] improved Hummer's methods by elimination $\mathrm{NaNO}_{3}$, increasing the concentration of $\mathrm{KMnO}_{4}$, and using a mixture of $\mathrm{H}_{2} \mathrm{SO}_{4} / \mathrm{H}_{3} \mathrm{PO}_{4}$, which obtained a high oxidized $\mathrm{GO}$ with free generation of toxic gases $\left(\mathrm{NO}_{x}\right)$. $\mathrm{rGO}$ can be obtained by chemical or thermal reduction treatments heated above $100{ }^{\circ} \mathrm{C}$ using reducing agents, such as hydrazine, hydrogen, etc. Li et al. [20] reduced GO dispersions to stable graphene colloids via hydrazine reduction under controlled conditions, which provided a simple method to prepare single-layer chemical converted graphene sheets with thickness of $\sim 1 \mathrm{~nm}$. Recently, Chhowalla's group [21] further simplified the method to reduce GO into $\mathrm{rGO}$ by using few seconds long microwave pulses. The microwave-reduced GO showed CVD graphene-like Raman features, as well as atomic-scale highly ordered structures.

\subsection{Graphene-based composites}

Graphene-based composites as filler materials where graphene is used as a base to disperse in or incorporation of other nanomaterials, such as metal nanostructures [22-24], semiconductor nanostructures [25-27], and polymers [28, 29]. Chemical reduction, hydrothermal method, microwave-assisted growth, solution mixing, in situ growth are often used to prepare graphene-based composites [30]. Kamat et al. [31] first decorated gold nanoparticles (AuNPs) by chemical reduction of $\mathrm{AuCl}^{4-}$ ions with $\mathrm{NaBH}_{4}$ on octadecylamine (ODA) modified graphene suspended in tetrahydrofuran (THF) medium. Gao et al. [32] reported the preparation of a graphene-CdS QD composite using a one-pot process, where simultaneously brought about the reduction of GO and the formation of CdS on graphene. Graphene-ploymer composites required polymer matrixes, such as poly-N-vinyl carbazole (PVK), poly(methyl methacrylate) (PMMA), Poly(L-lysine) (PLL), and polycaprolactone (PCL) [33].

\section{Graphene in biosystems: toxicology and bio- compatibility}

In this section, we first introduce studies on the interactions between graphene and biomacromolecules in extracellular environment. Then we summarize the progress on the cytotoxicity of graphene, how graphene enters cells, and how it affects cellular metabolism. Furthermore, we describe the in-vivo toxicity of graphene in various organs and model animals by demonstrating biodistribution of graphene, and their toxicological effects. Finally, we bring about an overview on the toxicology of graphene, and how we could circumvent this problem to realize real-world applications.

\subsection{Interactions between graphene and biomacromolecules}

\subsubsection{Interactions between graphene and nucleic acids}

It is well known that nucleic acids are essential in transmitting and expressing information through protein synthesis in living entities. Recently, a series of functional nucleic acids [34] are produced by a combinatorial method called in-vitro selection or systematic evolution of ligands by exponential enrichment (SELEX), functioning beyond the conventional genetic roles. The programmable pairing of DNA single strands into precisely engineered, connecting double helices make it an ideal material for assemblage of nano-architectures [35-39], such as DNA nanotube/nanowire [40], DNA array [41] and DNA origami [42-44], for advancing bioimaging [45-48], biomedicine $[49,50]$, and biomolecular sensing [51-53]. Thus, the study of interaction between graphene and nucleic acids is well appealing towards biomedical applications.

Up until now, some experimental studies regarding the absorption and desorption mechanisms between graphene and nucleic acids have been reported. Mann and co-workers [54] reported a method of utilizing single-stranded DNA (ssDNA) to prepare stable aqueous graphene suspensions sheets, which proposed a surface binding model for ssDNA/graphene interactions via non-covalent hydrophobic and electrostatic/hydrogen bonding between purine/pyrimidine bases of ssDNA and the carboxylic and phenolic groups of graphene. In general, the binding affinity between graphene and ssDNA via noncovalent interactions (i.e., $\pi-\pi$ stacking interactions and hydrophobic forces) is much higher than those between graphene and double-stranded DNA (dsDNA) or tertiary DNA structures. Chen and co-workers [55] demonstrated that GO could bind dyelabeled ssDNA and quench its fluorescence, where GO has lower affinity for dsDNA than for ssDNA. Fan et al. [56] developed a GO-based multicolor fluorescent DNA analysis and performed a molecular dynamic (MD) simulation to study the interaction differences between ssDNA and dsDNA with GO. As a result, the nucleobases of ssDNA lay nearly flat on the GO surface that led to the strong absorption by GO. Such interaction was ascribed to the $\pi-\pi$ stacking interactions between its nucleobases and hexagonal rings of GO. Whereas, the nucleobases of dsDNA in helical structure were shielded, so that GO could not stably adsorb it (Fig. 3(a)). Li's group [57] constructed graphene-based fluorescence resonance energy transfer (FRET) aptasensor for detection of thrombin by assembling FAM-labeled aptamer on graphene. When thrombin was introduced, it caused the conformational change of aptamer that induced the recovery of fluorescence (Fig. 3(b)). Liu and Maheshwari et al. [58] reported the binding efficiency of DNA to GO surface depends on DNA length, salt, $\mathrm{pH}$, solvent, and temperature. As reported, short DNA strands usually had faster kinetics and higher adsorption efficiency than long DNA strands. Also, higher salt concentration, lower $\mathrm{pH}$ value, and additional organic solvent facilitated the absorption of DNA on GO.

Kumar et al. [59] compared the interactions of DNA, polyamide (PNA) and polycarbamatenucleic acid (PCNA) oligomers with GO. They claimed that PNA and D-/L-PCNA possessed higher quenching efficiencies than that of DNA due to no generation of electrostatic charge repulsions. Yang et al. [60] reported that ssRNA showed similar adsorption and desorption abilities towards GO to ssDNA, as well as effective protection from enzymatic cleavage. Huang and co-workers [61] explored the size effect of nGO on ssDNA binding. They found that $\mathrm{nGO}$ sheets with $\sim 200 \mathrm{~nm}$ lateral dimensions had the highest quenching efficiency, while nGO sheets of $\sim 40 \mathrm{~nm}$ exhibited much weaker quenching efficiency (Fig. 3(c)).

Based upon the above findings, nucleic acid/graphene complexes have been used for DNA hybridization [62], DNA sequencing [63], protein identification [64], and cancer cell realization [65]. Fan and Hu et al. [64] used different DNA elements with high-precision identification for given analytes to set a small library of "ensemble aptamers" possessing "lock-and-key" recognition with molecular or cellular targets. Wang and Zhou et al. [62] determined the binding kinetics and affinity of DNA hybridization in real-time based on 
multi-channel graphene field-effect transistor (FET) sensors, where an analytical model was described for the evaluation of probe density, hybridization efficiency, and the maximum signal response.

In addition, some signal amplification strategies, such as rolling circulation amplification (RCA) [66, 67], hybridization chain reaction (HCR) [68], exonuclease III (Exo III)-triggered recycling of the targets $[69,70]$, have also been coupled to improve the properties of graphene/nucleic acids complexes. For example, Li's group [66] developed a graphene-based biosensing platform for ultrasensitive detection of thrombin and ATP based on rGO and rolling circulation amplification (RCA) (Fig. 3(d)). Fan's group [68] utilized GO as nanoprobes to investigate the interactions between GO and different DNA structures and real-time monitor HCR and RCA amplification process (Fig. 3(e)).

Apart from experimental studies, some theoretical researches have also been carried out regarding the binding mechanisms between graphene and nucleic acids. Gowtham et al. [71] investigated the interaction of nucleobases with graphene using density-functional theory (DFT) framework, finding the calculated binding energy in the order as $\mathrm{G}>\mathrm{A} \approx \mathrm{T} \approx \mathrm{C}>\mathrm{U}$. Rao and co-workers [72] optimized the structures of nucleobase/graphene and calculated binding energies following the sequence of $\mathrm{G}>\mathrm{A}>\mathrm{T}>\mathrm{C}$ by using the Jaguar computational package. Bhattacharyya et al. [73] reported ab initio quantum chemical calculations of graphene/nucleobase complexes by dispersion corrected DFT. It was found that large graphene sheets with curvatures might be more stable for nucleobase binding.

\subsubsection{Interactions between graphene and proteins}

Proteins are fundamental elements in all living organisms, which has physiological activities within cells. For nanomaterials to enter biological mediums, the first thing to encounter are the interaction with proteins (i.e., proteins, lipids, enzymes) [74-76]. Therefore, investigations of the interactions between graphene and proteins are essential for study the cellular uptake and biological toxicity of graphene.

Seo et al. [77] explored the adsorption topography on the surface of GO by atomic force microscopy (AFM). The antibody was particularly linked on the edges and folded structures of GO where were rich of carboxylic acid groups. Fan and Liu et al. [78] found that GO was an efficient quencher for fluorescent conjugated oligomer FBT with extensive $\alpha$-mannose side. In the presence of concanavalin A (ConA), the specific binding between FBT and ConA prevented GO/FBT interact which led to light-up sensing of lectin and Escherichia Coli (E. coli) (Fig. 3(f)). Dravid et al. [79] used nGO with $20 \mathrm{~nm}$ lateral dimensions as artificial receptor to identify 48 unknown proteins at concentrations of 100 and $10 \mathrm{nM}$. Liu and Peng et al. [80] studied the effects of PEGylation on serum behaviors of GO by using LC-MS/MS and Western blot analysis. They found PEGylated nGO (PEG-nGO) generated a nanointerface that significantly reduced serum protein binding and complement C3 activation. Niu and Chi et al. [81] investigated the interactions between stabilized chemical converted graphene (sCCG) and blood proteins. It is revealed that the sCCG/protein binds via the $\pi-\pi$
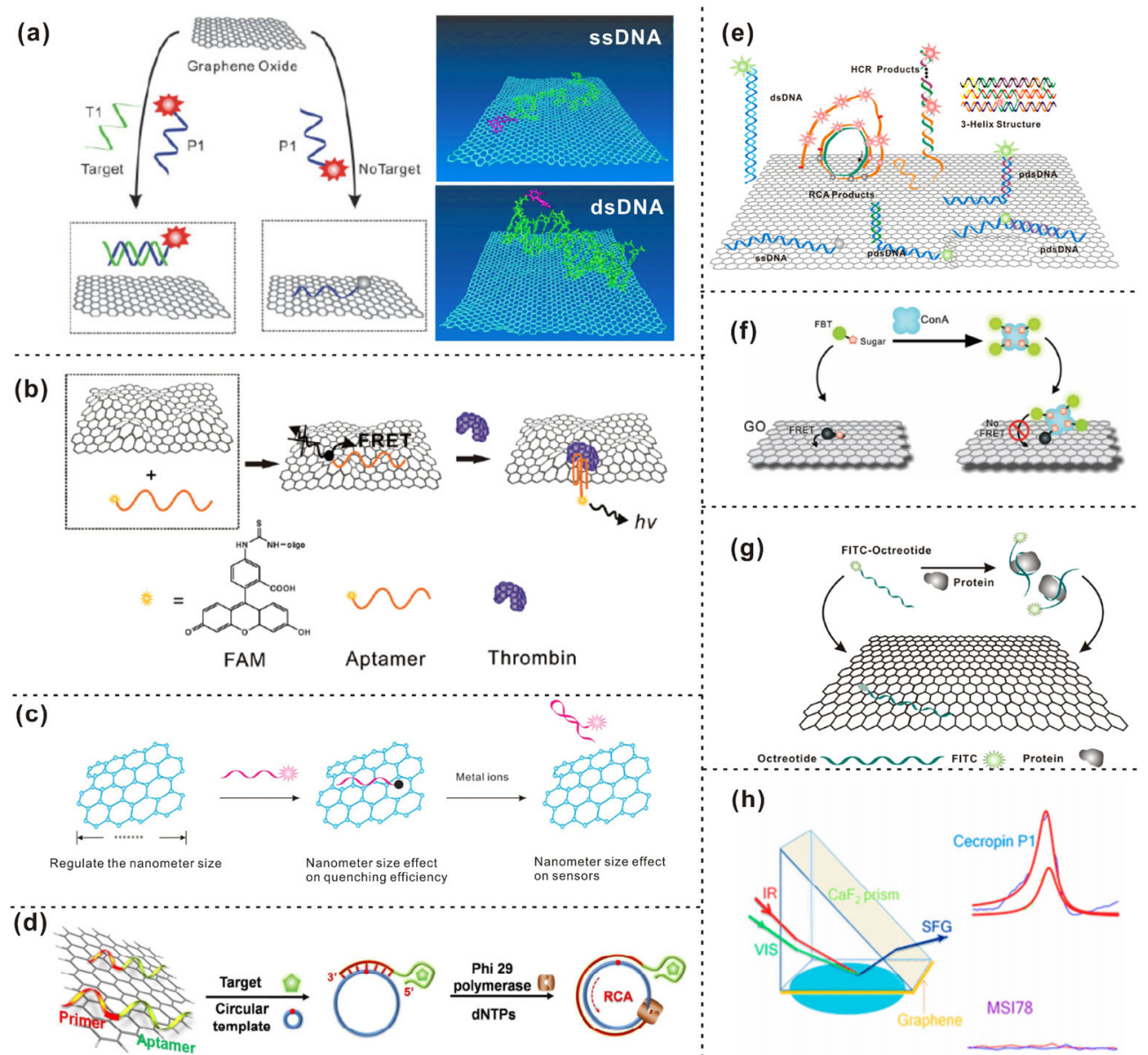

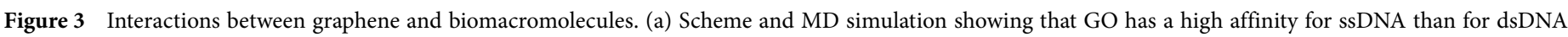

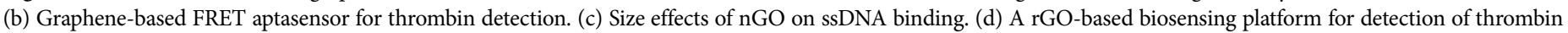

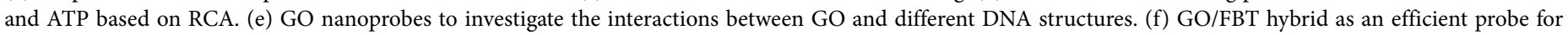

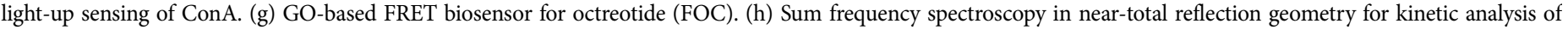

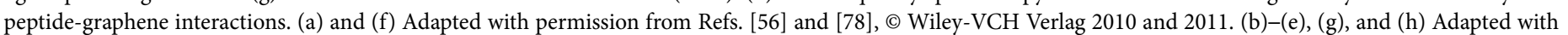
permission from Refs. [57], [61], [66], [68], [82], and [83], (c) American Chemical Society 2010, 2014, 2014, 2017, 2013, and 2017, respectively. 
interactions.

Investigation of the peptide-graphene interactions can be useful for understanding the protein functions within cells [82-84]. Huang et al. [82] developed a GO-based fluorescence resonance energy transfer (FRET)-biosensor using fluoresce in isothiocyanate (FITC)labeled octreotide (FOC) (Fig. 3(g)). As a result, FOC showed a high absorption affinity and high kinetics for GO via electrostatic forces and $\pi-\pi$ interactions, but a lower quenching efficiency of $78 \%$ than that for ssDNA. Very recently, Chen et al. [83] experimentally and theoretically studied the interactions between two peptides (cecropin P1 and MSI-78(C1)) and graphene via sum frequency generation vibrational spectroscopy and MD simulation (Fig. 3(h)). They found that the competition between planar and charged hydrophilic residues significantly affected the peptide-graphene interactions.

\subsection{Cellular studies of graphene}

\subsubsection{Graphene and viruses}

Some global infectious diseases that threat to human health have been caused by emerging and reemerging viruses, like Ebola, Zika, MERS-CoV and avian influenza virus. Owing to the superior physiochemical properties, graphene-based nanomaterials have been focused on the biological responses of viruses, including the capture and destruction, attachment inhibition, and detection of viruses. Min et al. [85] developed a multiplexed GO-based helicase assay (mGOHA) based on GO to screen viral enzymes: inhibitors of HCV NS3 helicase and severe acute respirator syndrome coronavirus (SARS CoV) helicase. As a result, 22 inhibitors of HCV NS3 helicase, 26 of SARS CoV helicase and 24 of both helicases was discovered from a 10,000 small molecule library. Wang and Tang et al. [86] demonstrated that GO could act as a label-free material for detection and disinfection of enteric viruses: EV71 and H9N2. GO can effectively interact with viruses, as well as significantly improve the destruction, removal and disinfection that achieved at least 6-log inactivation. Gedanken and Sarid et al. [87] prepared sulfonated magnetic nanoparticles functionalized reduced graphene oxide (SMRGO) to capture and photothermally destroy herpes simplex virus type 1 (HSV-1). They found that SMRGO could be served as an effective antiviral agent $(\sim 99.99 \%)$ with a short capture time of 7 min. Gadanken et al. [88] reported that HSV-1 infections were inhibited by GO and partially reduced sulfonated GO (rGO-SO3) via a competitive inhibition mechanism. Recently, Adeli and Haag et al. [89] synthesized highly active functionalized graphene sheets containing $6 \%$ graphene and $94 \%$ sulfated polyglycerol, which are able to trap 20 virions by individual sheets with the size of $\sim 1 \mu \mathrm{m} \times$ $1 \mu \mathrm{m}$.

Moreover, some graphene and its derivatives-based biosensing assays have been employed for analyzing viruses. For instance, colorimetric analysis of respiratory syncytial virus (RSV) using AuNP-GO hybrids [90], and graphene-FET biochip for Zika virus detection have been reported [91]. To obtain mechanically reinforced ultrathin membranes, Yoo and co-workers [92] developed a novel method to unidirectionally align M13 viruses on GO sheets in large area $\left(>20 \mathrm{~cm}^{2}\right)$ by applying shear force, in which the engineered peptide of STB1 fused to the end of the virus through salt-bridge forces. It was confirmed that the viral-nanomesh ultrathin membranes showed enhanced permeability and high size-selective exclusion.

\subsubsection{Graphene and mammalian cells}

Like other carbon nanomaterials (i.e., CNTs), graphene-based nanomaterials could also influence cell morphology, adhesion, differentiation, migration, proliferation and cytoskeleton organization. Graphene paper have provided an ideal platform for the growth and proliferation of the mouse fibroblast cell line (L-929) [93]. Likewise, graphene also serves as a preconcentration platform for osteogenic inducers for stem cell differentiation [94]. Studies also suggest that graphene-based nanomaterials treated by macromolecules exhibited good biocompatibility. Recent study [95] revealed that pristine graphene improved cell adhesion and proliferation, but it exhibits no influence on cell stress for both nonneuronal and neuronal cells, such as the mitochondrial membrane potential (MMP), the morphology and the autophagy levels. The functionalization of artificial peroxidase and extracellular matrix protein on graphene surface could improve the adhesion/growth capability of MCF-7 cells [96]. Both experimental observations and MD simulations showed that GO substrates treated by a mild thermal annealing process promoted the capture of Class II MHC-positive cell induced by oxygen clustering [97]. Besides acting as a good substrate, graphene has been designed as hybrid scaffold for tissue engineering applications. Fox example, the graphene-nanofiber hybrid scaffold by coating different amount of GO on the polymeric nanofiber (polycaprol actone) could cause selective differentiation of neural stem cells (NSCs), wherein a higher amount of GO facilitated cell differentiation into mature oligodendrocytes (Fig. 4(a)) [98]. Furthermore, a 3D GO/polymer hydrogel [99] served as an active cell scaffold for reversible cell capture triggered by near-infrared (NIR) light. Custom made 3D graphene micropatterns fabricated by a LightScribe DVD writer were used as scaffolds for guiding neuronal cell alignment [100].

Till now, there are conflicting conclusions on the cytotoxicity of graphene. Various cytotoxicity methods suggested GO could hardly enter A549 cells and has no obvious cytotoxicity in a dosedependent manner [101]. In vitro experiments revealed that GQD showed no obvious cytotoxicity against Hela cells because of its ultra-small size and high oxygen content [102]. Compared to single-walled carbon nanotube (SWCNT), graphene exhibited a low cytotoxic effect on PC12 cells in concentration- and shape-dependent manner [103]. However, another study indicated that GO could induce cytotoxicity and apoptosis in human lung cancer cells (BEAS-2B cell lines) [104]. Interestingly, when GO was coated with fetal bovine serum (FBS), it could greatly mitigate the cytotoxicity against A549 cells and eliminate cell membrane damage. It was also found that the cytotoxicity of FBS-coated GO showed little or no dependency on temperature (Fig. 4(b)) [105]. Similarly, reducing cytotoxicity of $\mathrm{GO} / \mathrm{rGO}$ mediated by blood proteins coating was also demonstrated by MD simulations [106].

The cytotoxicity of graphene exhibits concentration, size, shape and time dependency. The toxicity intensity was in accordance with increasing graphene concentration or incubation time [101, 103]. Ultrasmall GO nanosheet with lateral size less than $50 \mathrm{~nm}$ displayed lower cytotoxicity and higher cellular uptake than large sized GO nanosheets [107]. A comparative study [108] revealed that the cytotoxicity of graphene-based materials increased as the lateral size decreased and functional groups density increased, by assessing the interaction between GO, thermally rGO and chemically rGO and human lung cells (BEAS-2B and A549). On the contrary, another recent study found that large GO with micrometer size was more likely to damage cell membrane and cause pro-inflammatory response than small GO with nanometer size [109]. Besides that, surface oxidation state of graphene had a significant impact on the induction of cytotoxicity in mammalian cells and lung. Hydrated GO (hGO) with highest carbon radical led to much more lipid peroxidation and cell death in THP-1 and BEAS-2B cells. GO exhibited lesser effects, and rGO had minimal toxicity (Fig. 4(c)) [110].

\subsubsection{Signal pathways of graphene in cells}

It is revealed that the pristine graphene induced apoptosis in RAW 264.7 macrophages through the mitochondrial pathways [111]. Graphene exposure caused the depletion of the MMP and the 
(a)

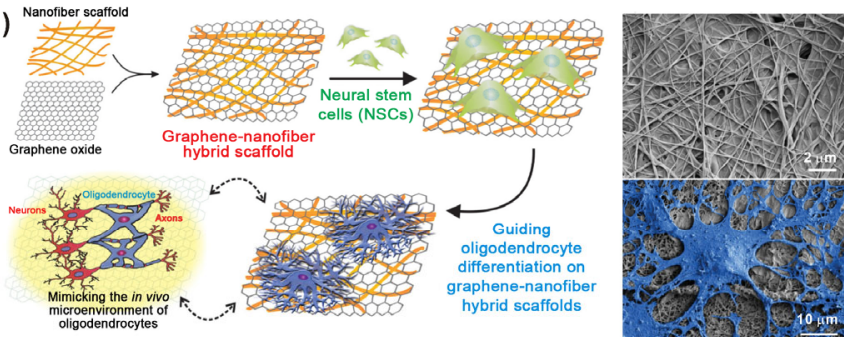

(b)

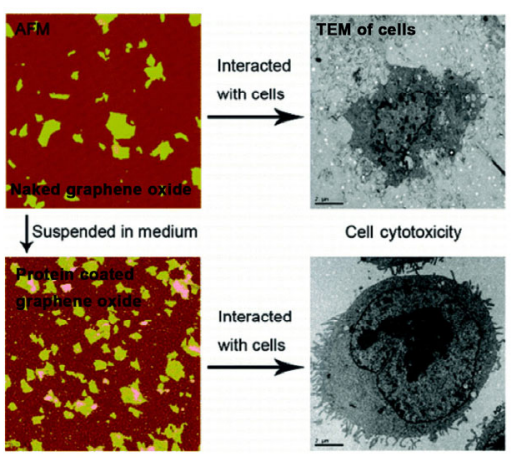

(c)

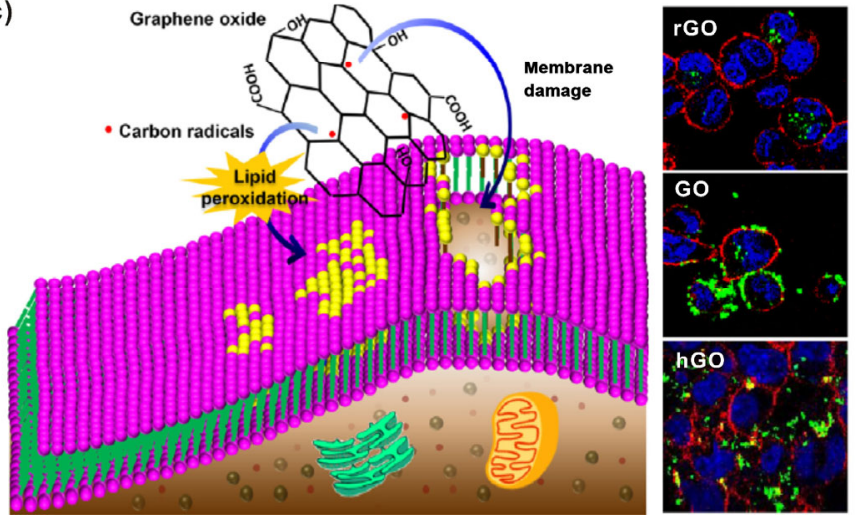

Figure 4 The role of graphene in cell growth and the cytotoxicity of graphene. (a) Left: scheme depicting the fabrication and application of graphene-nanofiber hybrid scaffolds; right: FE-SEM images of nanofibrous scaffolds (top) and cultured NSCs on the scaffolds (bottom). (b) AFM, TEM image and cell viability of A549 cells treated with GO nanosheets and FBS-coated GO nanosheets. (c) Left: membrane damage and lipid peroxidation in mammalian cells induced by carbon radicals of GO; right: confocal images of BEAS-2B cells after exposure to different GO samples. (a) Adapted with permission from Ref. [98], @ Wiley-VCH Verlag 2014 (b) and (c) Adapted with permission from Refs. [105] and [110], ( A American Chemical Society 2011 and 2018

accumulation of intracellular reactive oxygen species (ROS), leading to the activation of MAPKs- and TGF-beta signaling pathways which triggered Bim and Bax. Consequently, the caspase 3 were activated and the apoptosis was initiated. In another study [112], it was observed that GO treatment of cells simultaneously elicited autophagy [113] as well as TLR4/TLR9-regulated cytokine responses. The GO-induced autophagy was regulated by the TLRs signaling pathway which was associated with downstream adaptor proteins MyD88, TRIF and TRAF6. Furthermore, Mokhir et al. [114] proposed that surface-bound endoperoxide groups defined the capability of $\mathrm{GO}$ to enhance oxidative stress and cytotoxicity in Hela cells. Liu et al. [115] found that GO could induce plasma damages and cytoskeletal impairments in J774A.1 macrophages and A549 cells. Additionally, GO undermined the signaling transduction of integrin-FAK-RhoROCK pathway, resulting in plasma membrane perturbation. GO also affects the gene expression signature that was responsible for membrane and cytoskeletal damages.

A multiple-path mechanism has also been proposed to elucidate the stress-induced toxicity of GO/PP with Caenorhabditis elegans
(C. elegans) [116]. Under normal physiological conditions, cytochrome $\mathrm{c}$ (cyt c) was located on the inner membrane of mitochondria, and $\mathrm{H}_{2} \mathrm{O}_{2}$ was decomposed to $\cdot \mathrm{OH}$ catalyzed by GO/PP to a minimum which exerted no harmful effects on $C$. elegans. Under stress conditions, GO-mediated production of $\cdot \mathrm{OH}$ and cyt $\mathrm{c} / \mathrm{H}_{2} \mathrm{O}_{2}$ electron transfer may occur simultaneously. Since the over production of $\mathrm{H}_{2} \mathrm{O}_{2}$ was triggered by oxidative and thermal condition, the accelerating effects of GO/PP on $\cdot \mathrm{OH}$ generation were significantly improved. Moreover, cyt c provided chance for $\mathrm{GO} / \mathrm{PP}$ to direct interact with it. GO/PP elevated and accelerated the electron transfer between cyt $\mathrm{c}$ and $\mathrm{H}_{2} \mathrm{O}_{2}$, as well as destroyed the inherent antioxidant defense system, eventually leading to apparent toxicity to worms.

\subsection{In-vivo studies of graphene}

\subsubsection{Graphene in organs}

Some study [117] found that GO predominantly deposited in lung and induced pulmonary edema and granuloma formation after being injected intravenously at a high dosage of $10 \mathrm{mg} \cdot \mathrm{kg}^{-1}$ body weight (Fig. 5(a)), which showed relative longer blood circulation half time and lower uptake in reticuloendothelial system (RES) than other carbon nanomaterials. Surface modified graphene-based nanomaterials with improved dispersion and stability significantly mediated biodistribution and translocation of graphene in organs. For instance, PEGylated nGO mainly accumulated in liver and spleen that were excreted by renal and fecal clearance [118], in which the PEG coating could decrease the retention of nGO in RES and promote the clearance of nGO from the organs [119]. Similarly, GO-dextran (GO-DEX) conjugate was also trapped in RES organs including liver and spleen, which showed clearance from the blood without apparent short-term toxicity in treated mice [120]. PEGylated GO derivatives exhibited no clear tissue uptake via oral administration, but high accumulation in RES including liver and spleen after intraperitoneal injection [121]. Unlike PEGylated GO, PEGylated rGO was distributed on major organs such as liver, spleen, kidney and brain after intraperitoneal and intravenous administration. Repeated administration of PEGylated rGO could cause severe liver injury, congestion in kidney and enhanced proliferation of splenocytes (Fig. 5(b)) [122]. Moreover, a carboxylated photoluminescent GQD prepared from carbon fiber show no acute toxicity or morphological changes, most of which accumulated in liver, spleen, lung, kidney, and tumor sites at $24 \mathrm{~h}$ after intravenous injection [123]. Finally, GQDs did not induced accumulation in major organs possibly due to the metabolism through kidneys, as well as no obvious in vivo toxicities due to the ultrasmall size and high oxygen content of GQD [102].

\subsubsection{Graphene in animals}

The toxic effects of graphene to animals have also been investigated [124]. In vivo toxicological study revealed that multifunctional graphene with fluorescence and magnetic properties did not cause any significant abnormalities nor influence the survival rate of zebrafish. In vivo whole-animal imaging confirmed that multifunctional graphene co-localized and evenly biodistributed from the head to tail in zebrafish [125]. The toxicity of GO and PEGylated polyL-lysine (GO/PP) functionalized GO were explored under stress conditions using a simple animal model, C. elegans worm. The results showed that GO/PP facilitated the electron transfer that impaired the inherent antioxidant defense system, and eventually triggered nanotoxicity to worms [116]. Graphene- $\mathrm{TiO}_{2}$ composite film could inactivate the $C$. elegans nematodes under solar light irradiation with concentration-dependency, implying the application in destruction of minuscule animals on biological environments [126]. 
(a)

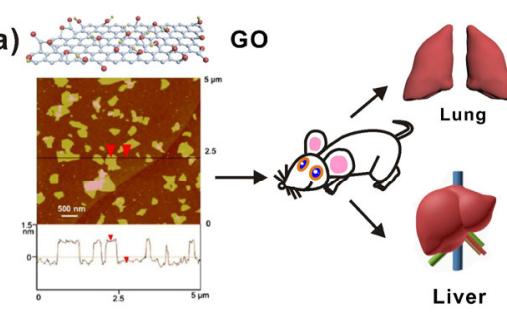

(b)

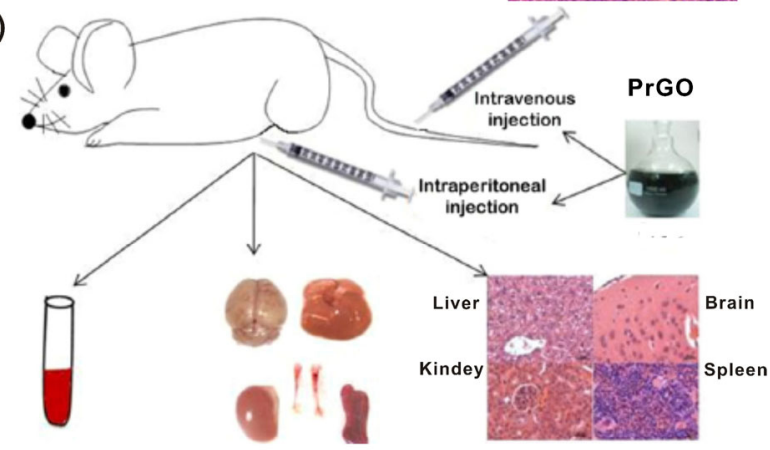

(c)

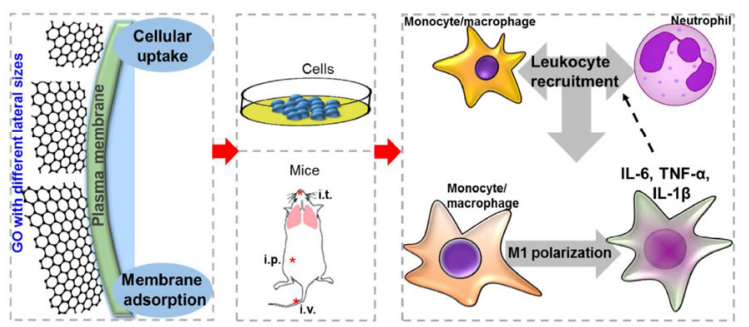

Figure 5 In vivo studies of graphene. (a) In vivo biodistribution of GO at a high dosage $(10 \mathrm{mg} / \mathrm{kg}$ body weight) in male mice after a single intravenous injection. (b) In vivo biodistribution of $\mathrm{PrGO}$ in mice after intravenous and intraperitoneal injection. (c) GO induced inflammatory responses in a size-dependent manner. (a) and (b) Adapted with permission from Refs. [117] and [122], () Elsevier B.V. 2011 and 2017. (c) Adapted with permission from Ref. [128], () American Chemical Society 2015.

Long-term toxicity of rGO nanosheets on female mouse reproductive performance and offspring development was investigated [127]. The results showed that $\mathrm{rGO}$ did not significantly change the sex hormone level of adult female mice. After intravenous injection, mouse dams achieved normal pregnancies and produce healthy offsprings before pregnancy or at an early gestational stage, whereas all surviving mice had abortions if they were injected with low or medium doses at a late stage of gestational. Besides toxic effects on animals, GO demonstrated lateral size-dependent effects in the induction of inflammatory responses in mice [128]. Larger GO sheets showed a plasma membrane adsorption and gave more production of inflammatory cytokines and recruitment of immune cells, whereas smaller $\mathrm{GO}$ sheets were prone to be taken up by macrophages (Fig. 5(c)).

\subsubsection{Graphene in plants - phytotoxicity}

Graphene showed toxic effects on terrestrial plants of cabbage, tomato, and red spinach in a concentration-dependent manner, resulting in significant growth inhibition and biomass decrease mediated by the overproduction of ROS [129]. Likewise, GO demonstrated negative effects on the growth of wheat plants, which significantly caused root elongation, impairment of hair production, inhibition of photosynthesis and imbalance of nutrient homeostasis after 30 days of culture [130]. Interestingly, under normal levels, GO exposure did not induce obvious toxicity on Arabidopsis plants in seed germination, shoot and root development of seedlings, and flowering time. GO did not accumulated in mesophyll and parenchyma cells of lead or stem, but in root hair and parenchyma cells $[131,132]$. However, under drought or salt stress conditions, it influenced the development of the seedlings, and induced the translocation of GO from the roots to the leaves [131]. In another study, it was found that GO accumulated in the root at $112 \mathrm{~g} / \mathrm{g}$ after 15 days using ${ }^{13} \mathrm{C}$-stable labeling of the $\mathrm{C}$-skeleton, which inhibited the growth of wheat, altered root structure and cellular ultrastructure [133].

\subsection{Toxicological concerns of graphene}

Similar to other inorganic nanomaterials, graphene has also been challenged by toxicological concerns in regard to its potential biomedical applications. There are several inevitable issues should be taken into account in graphene toxicology, such as valid amount, toxicity criteria, irreversible/reversible toxic effect, and joint toxicity. First of all, the target dose is a direct and valid concentration in the biological response studies in the detailed experiments; unfortunately, numerous studies have used exposure dose to represent concentration-bioresponse relationships, which resulted in a false relevance for cytotoxic effects. On the other hand, current acceptance criteria for toxicity tests are unknown. In spite of certain inconsistencies in some experimental results and hypotheses of the toxicity mechanisms, a wealth of reports agree that physicochemical properties of graphene-based nanomaterials significantly affect the toxicological responses, such as sizes, charges, coatings, and structural defects of graphene. To compare the nanotoxicity obtained under different conditions, an acceptance criterion for toxicity tests, including choice of statistical assumptions, statistical procedures, or set of experimental conditions, is necessary. Also, a long-term test upon multiple administrations to evaluate the risks of graphene is lacking. Despite many studies reported in vivo behaviors, the irreversible/reversible toxic effects of graphene are unclear because the acute toxic assays often ignored the reversible or delayed effects upon target organs. Last but not least, the literatures have been reported that joint toxicity is greatly different from single toxicity $[134,135]$. Synergistic effects can occur between metal ions, organic materials, or biomolecules with graphene. And so the concern of joint toxicity should be paid enough attention, avoiding overestimation or underestimation the graphene toxicity.

\section{Applications}

Based on their inherent antibacterial properties, as well as superior physiochemical properties, graphene-based nanomaterials have been employed as a novel and green antibacterial material, as well as other applications in the fields of wound addressing, tissue engineering scaffolds, drug delivery, and water purification. In this section, we will give an overview of recent achievements and advancements wherein graphene-based nanocomposites have been applied.

\subsection{Antibacterial applications of graphene-based nano- materials}

Bacterial infection is one of the worldwide public health concerns, particularly in poor regions and income countries. Despite antibiotics are widely used as effective antibacterial agent, bacterial resistance makes it difficult to therapy the infection. To address this issue, novel antibacterial agents, like CNTs, metal/metal oxide NPs, have been investigated. In recent years, due to the inherent bacterial toxicity and negligible cytotoxicity, considerable efforts have been devoted to exploring antimicrobial activities of graphene-based nanomaterials, involving two forms: pristine graphene family and graphene-based composites.

\subsubsection{Antibacterial activities of graphene and its derivatives}

Pristine graphene family mainly contains graphene, GO and rGO, 
whose antibacterial activities are caused by physical damages or chemical effects. Huang and Fan et al. [136] first reported the antibacterial activity of GO and rGO nanosheets (Fig. 6(a)). After treatment with $85 \mathrm{~g} / \mathrm{mL} \mathrm{GO} / \mathrm{rGO}$ for $2 \mathrm{~h}$, the growth of $E$. coli was almost completely suppressed, with a viability loss up to $>90 \%$. Moreover, macroscopic freestanding GO/rGO paper made by vacuum filtration also exhibited the superior antibacterial effect, which could open up new opportunities for developing antibacterial material. Akhavan et al. [137] studied the bacterial toxicity of graphene nanowalls (GONWs) prepared by electrophoretic deposition (EPD) and reduced graphene nanowalls (RGNWs) obtained by hydrazine reduction. Both GONWs and RGNWs exhibited bacterial cytotoxicity to Gram-negative E. Coli bacteria and Gram-positive Staphylococcus aureus (S. aureus) bacteria. In particular, RGNWs exhibited higher antibacterial activity than GONW because of more sharp edges of the nanowalls leading to more cell membrane damage of the bacteria (Fig. 6(b)). Chen and co-workers [138] compared the bacterial toxicity of graphite (Gt), graphite oxide (GtO), GO and rGO using E. coli as a bacterial model. Under the same conditions, GO exhibited the highest antibacterial activities, followed by rGO, graphite, and GtO (Fig. 6(c)). Besides the antibacterial activities described above, the investigations of GO against dental pathogens has also been reported by Tang et al. [139]. Three typical dental bacterial, including $S$. mutans, $P$. gingivalis and F. nucleatum, were chosen to estimate the bacterial toxicity of nGO sheets. It was clearly confirmed that GO showed prominent antibacterial effect toward dental bacterial by using 3-(4,5-dimethylthiazol-2-yl)-2,5-diphenyl tetrazolium bromide (MTT) reduced assay, colony forming units (a)
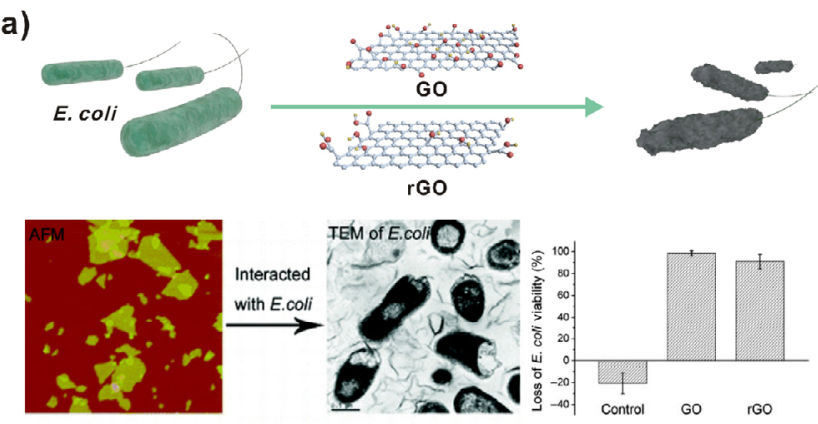

Graphene oxide sheets

(up) and paper (down)
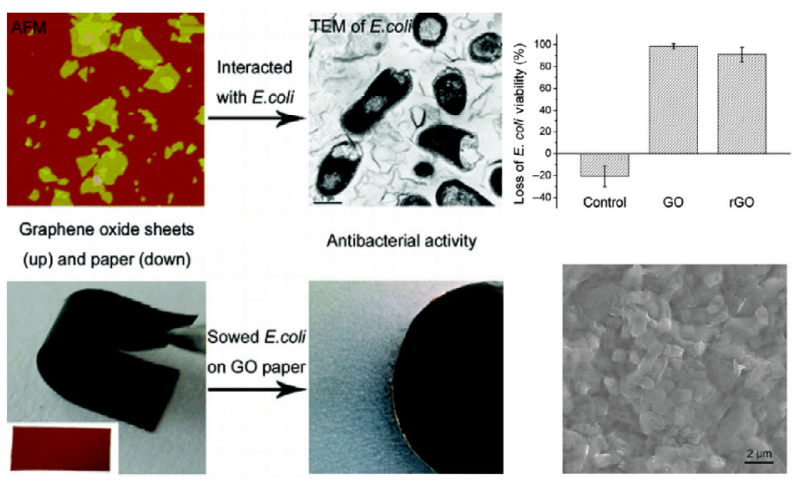

rGo
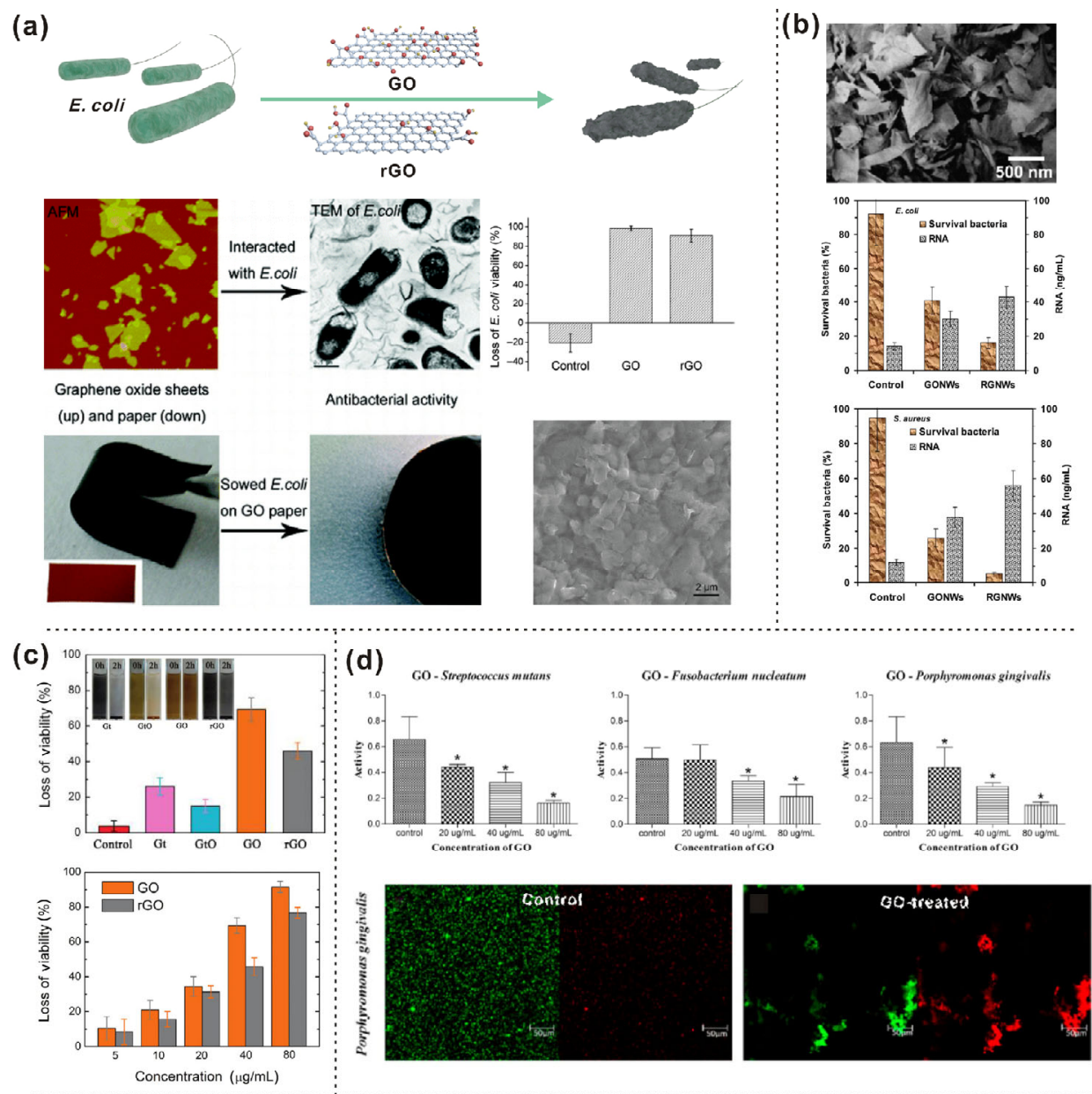

(e)
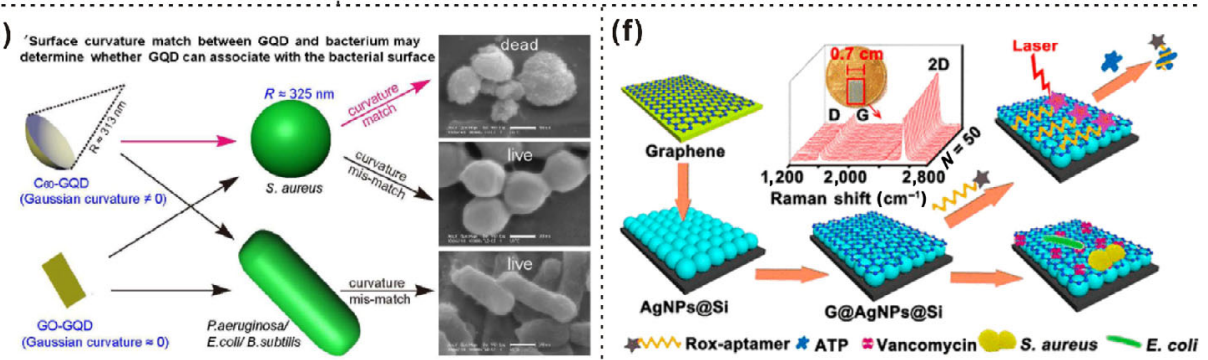

Figure 6 Antibacterial activities of graphene-based nanomaterials. (a) Antibacterial activities of GO and rGO sheets and papers. Top: schematics showing antibacterial activities of GO and rGO against E. coli; middle: AFM image of GO nanosheets, TEM image of E. coli treated with GO nanosheets, and loss of E. coli cell viability incubated with GO and rGO nanosheets; bottom: photograph of freestanding GO paper, and photograph and SEM image of $E$. coli growth on GO paper. (b) SEM image of GONWs prepared by EPD, and cytotoxicity of GONWs and RGNWs against E. coli and S. aureus. (c) Top: antibacterial activity graphene-based nanomaterials $(80 \mu \mathrm{g} / \mathrm{mL})$ to E. coli; bottom: different concentrations of GO and rGO after incubation with E. coli for $2 \mathrm{~h}$. (d) Top: antibacterial activities of GO to three typical dental bacterial evaluated by MTT assay; bottom: live/dead fluorescent staining images showing that after $2 \mathrm{~h}$ treatment with isotonic saline (control) and GO of $P$. gingivalis cells. (e) A schematic representation of how surface curvature match affect the GQD's antimicrobial activities. (f) Schematic illustration of fabrication of graphene-AgNPs-Si nanohybrids as a SERS platform for molecular detection to bacterial capture, discrimination, and inactivation. (a)-(f) Adapted with permission from Refs. [136], [137], [138], [139], [143], and [153], ( ) American Chemical Society 2010, 2010, 2011, 2015, 2016 and 2018, respectively. 
(CFU) counting, growth curve observation, and fluorescent live/dead staining (Fig. 6(d)).

Many factors related to the physiochemical properties of graphenebased material, such as lateral dimension, surface area, layer number, surface chemistry, and shape, can influence their antimicrobial effects. The size of GO sheet was a main factor influencing graphene's antibacterial activity. GO sheet with a larger size induced higher antibacterial activity than do smaller one $[140,141]$, either induced by direct insertion or cutting $[137,141]$ or lipid extraction of phospholipids [141] on treatment with graphene nanosheets. On the contrary, Elimelech et al. found that the cell inactivation of GO with surface coatings increased by 4 times as the average area of GO decreased from 0.65 to $0.01 \mathrm{~m}^{2}$. It was mediated via oxidative mechanisms because of intensive defects in smaller GO sheets [142]. The source material and bacterial shape were also associated with antibacterial properties of graphene. It was reported that [143] GQDs prepared from GO sheets (GO-GQDs) did not show antibacterial ability, whereas GQDs synthesized by rupturing $\mathrm{C}_{60}$ cage ( $\mathrm{C}_{60}$-GQDs) could effectively eliminate $S$. aureus, but not other three bacteria, Bacillus subtilis (B. subtilis), E. coli, and Pseudomonas aeruginosa ( $P$. aeruginosa). It seemed that surface Gaussian-curvature match between a GQD and a bacterium membrane might determine whether GQD cause the damaging the integrity of bacterial cell envelope (Fig. 6(e)). The doping of nitrogen (N) was considered to be a useful method to improve the properties of GQDs. A recent study showed that N-doped GQD could generate more ROS than $\mathrm{N}$-free GQD, implying enhanced antibacterial activities [144]. Other than the intrinsic properties of graphene-based nanomaterials, studies also revealed GO killed bacteria in concentration- and time-dependent manners [140].

\subsubsection{Antibacterial activities of graphene-based composites}

It is well known that silver acts as an effective antibacterial agent for centuries. $\mathrm{Ag}^{+}$treatment resulted in the detachment of cytoplasm membrane from the cell wall for $E$. coli and $S$. aureus and inactivated DNA replication and protein [145]. Gelatinous AgNPs with a diameter less than $10 \mathrm{~nm}$ effectively killed different Gram-positive and Gram-negative bacteria. Unfortunately, bare AgNPs aggregated when they directly interact with bacteria. To alleviate this issue, Ag-graphene nanocomposites have been synthesized via different reducing agents, like $\mathrm{NaBH}_{4}$ [146], hydrazine [138], and dopamine [147], etc. As-prepared Ag-rGO nanohybrid displayed surface enhanced Raman scattering (SERS) and high disinfection activities [148, 149]. Ag-rGO nanocomposites also could be prepared without additional reducing agents, which showed enhanced antibacterial ability likely caused by the synergistic effect of "capturing-killing process", but no tolerable edema and/or erythema in skin irritation test [150]. Tan et al. develop a new method by using DNA as a template to prepare metal NPs and GO composites with controllable size and shape [151, 152]. Among the as-synthesized nanocomposites, $20 \mathrm{ppm}$ Ag(18nm)@dsDNA@GO and 16 ppm Ag(5nm)@dsDNA@GO composites showed optimal antimicrobial capability towards a model plant bacterium Xanthomon asperforans due to the synergistic effect between AgNPs and GO [151]. He et al. prepared grapheneAgNPs-Si nanohybrids (G@AgNPs@Si) as a multifunctional SERS platform, allowing for molecular to cellular detection. In particular, it was utilized for capturing, discriminating, and inactivating of E. coli and S. aureus bacteria with strong antibacterial rate of $\sim 93 \%$ after incubation for $24 \mathrm{~h}$ (Fig. 6(f)) [153]. In addition, copper, gold and lanthanum has also been anchored on graphene to kill bacteria [154-156].

Titanium oxide $\left(\mathrm{TiO}_{2}\right)$ is an important and inexpensive photocatalytic material. The $\mathrm{rGO} / \mathrm{TiO}_{2}$ thin films as photocatalysts degraded E. coli bacterial under solar light irradiation. The reduction of $\mathrm{GO}$ for $4 \mathrm{~h}$ maximally improved the antibacterial effect of bare $\mathrm{TiO}_{2}$ films by approximate 7.5-fold [157]. $\mathrm{GO}-\mathrm{TiO}_{2}$ nanorod composites (NRCs) on the gram scale exhibited higher antibacterial activities against $E$. coli under simulated solar light irradiation than that of $\mathrm{TiO}_{2}$ NRCs, which was ascribed to the anti-charge recombination and increased (101) facets [158]. The flexible hybrid films comprising of free standing graphene strongly coupled with Ti nanosheets showed almost complete sterilization of $E$. coli within only $15 \min$ [159].

The poor dispersibility and processability of graphene limit its application in bacteria disinfection. To address these challenges, dispersible polymer-graphene hybrids have been formed via $\pi-\pi$ stacking interaction formed between them by embedding graphene into polymer matrix. Rodrigues and co-workers [160] fabricated an antimicrobial film containing PVK polymer and GO on ITO surface. The resulting film was $90 \%$ more effective in inactivating $E$. coli bacterial colonization, in which the inclusion of PVK led to the enhanced bacterial toxicity. PLL as a polycationic homopolymer facilitates the attachment and immobilization cells on solid substrate. Lee et al. [161] prepared various graphene-PLL composites through electrostatic interactions and covalent bonding between PLL and $\mathrm{GO} / \mathrm{rGO}$ sheets. Among the as-prepared composites, grapheneDS-PLL composed of 4-carboxylic acid benzene diazonium salt (DS) showed the most effective antibacterial behavior given the production of carboxylic acid groups, while improving the growth of human cell.

\subsubsection{Mechanisms of antibacterial activities of graphene-based nanomaterials}

Despite increasing number of experimental results, however, there is no identical mechanism on how graphene-based nanomaterials cause bacterial inactivation, which may be related to the inconsistent physicochemical properties of graphene-based nanomaterials and experimental surroundings. According to recent achievements, several predominant mechanisms have been proposed to explain the graphene-based nanomaterial's antimicrobial behavior, that is, the membrane damage hypothesis, the oxidative stress hypothesis, the wrapping or trapping hypothesis, and other mechanisms.

The membrane damage hypothesis. Several studies have demonstrated that the sharp edges of graphene-based nanomaterials, also called "nano-knives", "cutters", or "blades" could penetrate into and physically damage the cell membrane of bacteria, resulting in the leakage of intracellular materials and eventual cell death. The mechanism is sometimes mentioned as insertion or penetration mode, which could induce membrane stress and thus inactive microorganisms documented in experimental studies and theoretical simulations.

Akhavan and Ghaderi [137] first proposed that the bacterial cell membrane was effectively damaged by direct contact interaction with extremely sharp edges of the NWs, and subsequently leading to leakage of RNA and losing of cell vitality. Tu et al. [141] experimentally and theoretically studied how graphene and GO nanosheets cause the damage of $E$. coli within the outer and inner membranes. In the TEM experiments and MD simulations, it was both clearly observed two kinds of molecular mechanism: direct insertion/cutting mode and destructive lipid extraction mode (Fig. 7). The vigorous extraction of phospholipids from the lipid bilayers was first discovered in MD simulations, which was further verified by typical TEM images. The strong attraction between graphene and lipid molecules was mostly generated from unique $2 \mathrm{D}$ carbons with $\mathrm{sp}^{2}$-bonded structures, promoting unexpected strong dispersion interactions between them. Both insertion and extraction could cause severe membrane stress, leading to the degradation of the bacterial membrane and then the reduction of bacterial viability.

Similarly, the two basic modes of insertion/cutting and lipid 

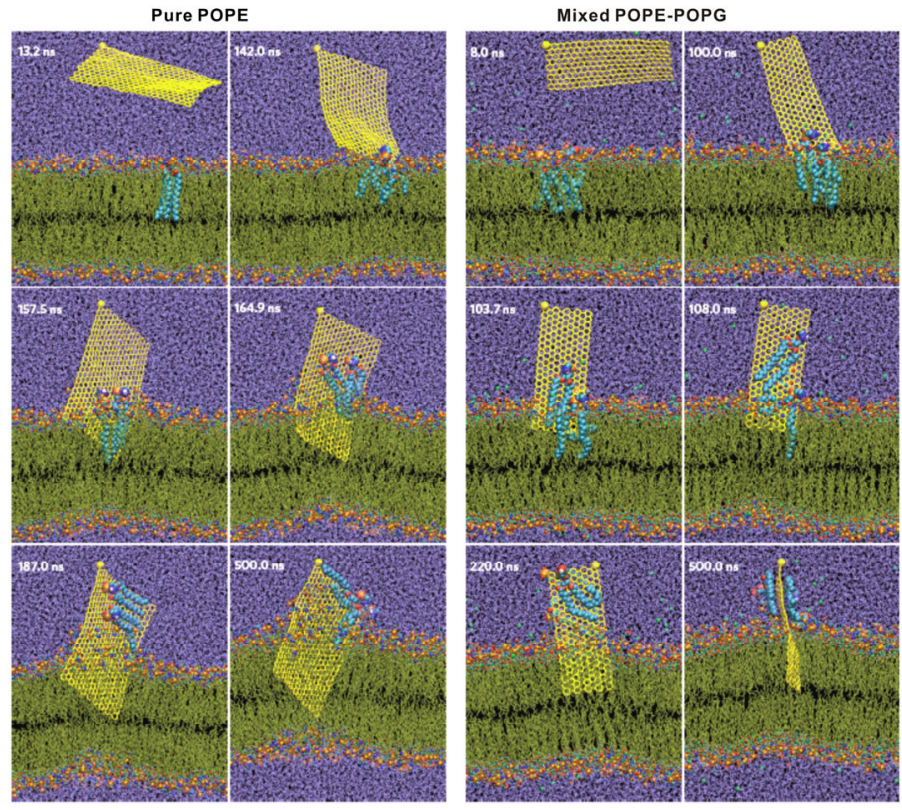

Figure 7 Two representative trajectories for graphene nanosheets insertion and lipid extraction for outer membrane (pure POPE) and inner membrane (3:1 mixed POPE-POPG). Adapted with permission from Ref.[141], (c) Nature Publishing Group 2013.

extraction were induced by blade-like graphene-based nanomaterials, which were also observed in other studies. Liu et al. [138] presented that direct contact with graphene nanosheets could disrupt or damage cell membrane, where sharp edge of graphene sheets acted as "cutters" and caused membrane stress, previously applicable for SWCNTs. Li et al. [162] revealed that few-layered graphene flake started with Brownian motion, followed by spontaneous piercing of the membrane with its sharp corners or protrusions at edges, eventually achieving complete penetration into the membranes. While the previous studies have revealed that graphene surfaces with an orientation of $90^{\circ}[136,138,140]$ were effective for cell inactivation, Pham et al. [163] found that graphene surfaces with a $37^{\circ}$ orientation has the best efficiency to kill bacteria. It is also found that the density of graphene edges served as a crucial parameter in the determination of antimicrobial behavior.

Moreover, some theoretical analysis has proposed the nearperpendicular penetration as another interaction mode between graphene and cell membrane. Yi and Gao [164] found that graphene sheets in a size-dependent manner would adopt a near-perpendicular configuration or a parallel attachment onto a membrane surface, where the membrane splay and tension energies acted as the main driving force. The inconsistent modes, however, were proposed by Dallavelle et al. [165]. They reported that smaller graphene sheets were more freely to pierce through transmembrane, while larger ones lay mainly flat on the surface of bilayers where wreak havoc was created with lipophilic membranes and formed a patch of upturned phospholipids with their tails direct contact with the graphene sheet.

However, some other findings $[166,167]$ proposed different antibacterial mechanism that the basal planes of GO may determine its bacterial disinfection. Advincula et al. [166] found that GO sheets entirely deposited by Langmuir-Blodgett (LB) film still retained the antibacterial activity, exhibiting positive correlation between the number of LB layers and bacterial inactivation. In another study [167], when bovine serum albumin (BSA) or tryptophan (Trp) molecules were noncovalently adsorbed on GO basal planes, the occupation could cause GO's deactivation against bacteria, suggesting the availability of GO basal planes in the cytotoxicity against bacteria. Recently, Haag et al. [168] confirmed that the antimicrobial property of graphene was very relevant to both its sharp edges, basal planes and tight agglomeration by studying the interactions between functionalized graphene derivatives and bacteria.

The oxidative stress hypothesis. Graphene-induced oxidative stress has been proposed as another widely recognized mechanism for antimicrobial activities of graphene-based nanomaterials, which is comparable to that of other carbon-based materials, such as CNTs and fullerene with similar structural and physiochemical properties $[103,169,170]$. Generally, there are two pathways for the production of oxidative stress: ROS-dependent and ROS-independent mechanism. The former arises from the elevated intracellular ROS levels, while the latter brings about damage or oxidation of cellular structure through electron transfer. Oxidative stress can disturb microbial metabolism and destroy cellular functions, resulting in the loss of cell integrity and eventually leading to cell death.

ROS-dependent pathway occurs via the excess accumulation of ROS, including hydrogen peroxide $\left(\mathrm{H}_{2} \mathrm{O}_{2}\right)$, hydroxyl radicals $\left(\mathrm{OH}^{\bullet}\right)$, superoxide anions $\left(\mathrm{O}^{-{ }^{-}}\right)$, or singlet molecular oxygen $\left({ }^{1} \mathrm{O} 2\right)$. The elevated ROS level makes cell reach an oxidative state, and further lead to the damage or inactivation to cellular substrates, e.g., proteins, DNA, and lipids, followed by dysfunction of the mitochondria, disintegration of cell membrane, and ultimate cell death by apoptosis or necrosis [171]. In the ROS-dependent pathway, antioxidant compounds, like glutathione (GSH), N-acetylcysteine (NAC) [172], a-tocopherol [173], and dichlorodihydrofluorescein diacetate [103, 174] can be oxidized in the presence of ROS and act as intracellular redox state indicator, whose depletion suggests the antibacterial effect of oxidative stress against bacteria. Kim's group [172] measured the levels of ROS in $P$. aeruginosa cells treated by GO and rGO, which were 3.8- and 2.7-fold higher than that in untreated cells, respectively. It was demonstrated that pretreated cells with the antioxidant GSH or NAC reduced the levels of ROS derived from GO or rGO, implying that cell death is mediated with ROS generation. They further observed a remarkable higher production of $\mathrm{O} 2^{*-}$ by $\mathrm{GO}$ or rGO, directly verifying the toxicity of ROS against $E$. coli [175].

Lipid peroxidation is an important oxidative pathway initiated by ROS-mediated oxidation of lipid molecules, which proceeds by a free radical chain reaction mechanism. In lipid peroxidation, lipid peroxide radicals are further formed to propagate the oxidative damage through the membrane. Kim and co-workers [176] found the ultrasound-induced lipid peroxidation was enhanced by $117 \%$ and $109 \%$ after treatment with 10 and $5 \mu \mathrm{g} \cdot \mathrm{mL}^{-1}$ of graphene, respectively. Elimelech et al. [142] reported that preincubated bacterial cells with a lipid-soluble antioxidant $a$-tocopherol, reduced the antimicrobial effect of GO sheets, indicating that oxidative stress served as a crucial factor in GO-induced cell inactivation. Moreover, the cytotoxicity of graphene was proven to be effected by mitochondrial membrane depolarization. Chen et al. [111] evaluated the cytotoxicity and the related signaling pathways of graphene in murine RAW 264.7 macrophages. After exposure of cells to graphene, the inactivation of bacteria is generated by the loss of MMP and the accumulation of ROS, followed by triggering apoptosis through activation of the mitochondrial pathway.

Besides ROS-mediated damage, the other possible pathway is ROS-independent oxidative stress against bacteria, in which antimicrobial behaviors stem from graphene by disruption or oxidation of cell architecture or component without ROS production. This behavior was previously observed in fullerene $\left(\mathrm{C}_{60}\right)$-exposed bacteria, where $\mathrm{C}_{60}$ acted as an oxidant and exerted ROS-independent oxidative stress against bacteria [169, 177]. Liu et al. [138] observed no detectable levels of $\mathrm{O}^{*-}$ in antibacterial activity, indicating that graphene-based nanomaterials mediated little ROS production. They later used Ellman's assay to evaluate the oxidation of GSH and indirectly verified that graphene-based nanomaterials possessed the capacity of mediating ROS-independent oxidative stress against 


\section{bacteria.}

Li et al. [178] represented a controversial view that the antibacterial activity of graphene does not arise from ROS-dependent damage but through electron transfer interaction between cell membrane and graphene. They found that graphene films deposit on $\mathrm{Cu}$ and Ge could inhibit the bacterial growth, whereas no obvious membrane damage was observed by graphene on $\mathrm{SiO}_{2}$. An electron transfer theory was proposed to explain the phenomena. A circuit for electron transfer was formed, where the electrons were easily transferred from bacterial membrane to graphene film and then to the conductor $\mathrm{Cu}$ or semiconductor $\mathrm{Ge}$ substrate, while the electrons could not be transferred to insulator $\mathrm{SiO}_{2}$ substrate. Consequently, the graphene-on-substrate junctions could serve as an electron pump for both $\mathrm{Cu}$ and $\mathrm{Ge}$ substrates, in which the electrons are quickly and potently extracted away from the bacterial membrane under the negative membrane potential, eventually leading to the destroy of membrane integrity and the loss of cell viability.

The wrapping or trapping hypothesis. Owing to its unique flexible 2D lateral nanostructure and the thinnest film, wrapping or trapping bacterial membranes has been regarded as the third mechanism of the antibacterial action. Rodrigues's group [179] found PVK-GO nanocomposite displayed excellent antibacterial behaviors through encapsulating the bacterial cells, resulting in reduced metabolic activity and inhibited bacterial growth. With slight difference, some researchers found that wrapping of bacteria could partly cause bacterial structural damage. Han's group [180] demonstrated that GO interwound or enveloped pathogens using an intact and relatively smooth cell wall or membrane. They further observed MMP changes and thus confirmed that GO could induce partly structural damages of pathogens via membrane depolarization, finally causing the bacterial death. Similarly, another study [181] found that 3D porous GO membrane could kill $S$. aureus through mechanical wrapping, disrupting and damaging cell membranes, and ultimate cell lysis. Akhavan et al. [182] proposed E. coli chemically reduced GO sheet could trap within the aggregated sheets, which biologically disconnected the bacteria from suspension and inactivated their proliferation.

Other mechanisms. Besides the mechanisms described above, there are some other viewpoints have been established, including extraction of lipid bilayers, interference of protein-protein interactions, etc. Recent experiments and simulations [141] showed that graphene's $\mathrm{sp}^{2}$ hybridized 2D carbon architecture has strong interaction with membrane lipids. As a consequence, graphene nanosheets could vigorously extract large amounts of phospholipids from the lipid bilayers, causing a deformation of the membrane and thus leading to the loss of membrane integrity. Luan et al. used large-scale all-atom MD simulations [183] to study the potential toxicity of graphene to cell functions. They found the insertion of graphene into the protein-protein interface could destabilize hydrophobic protein-protein interaction. The separated protein complexes destroyed cell's function and induced ultimate cell death, which agreed well with previous findings of the genotoxicity of CNTs to DNA [103].

\subsection{Other applications}

\subsubsection{Wound addressing}

If skins are damaged, microbes are liable to invade easily and formed severe wound infection. Commercially available wound dressing, like cotton wool, natural or synthetic bandages and gauzes, is important for an early stage of wound healing; however, they easily adhere onto wound surfaces and cause trauma to the healing wound. To overcome this issue, graphene-based nanocomposites in the form of fiber or hydrogel have recently emerged as promising candidates for wound dressing applications.
Fan's group [184] prepared GO-based antibacterial cotton fabrics via three ways, including direct adsorption, radiation-induced crosslinking, and chemical crosslinking. These GO-containing fabrics exhibited strong antibacterial behaviors and could kill $98 \%$ of bacteria (Fig. 8(a)). Also importantly, these Cotton-GO fabrics could keep a high inactivation efficiency of more than $90 \%$ even after being washed 100 times, as well as cause no irritation to wounded skin. The flexible, foldable and reusable cotton-GO fabrics provided a good prospect for potential antibacterial applications. Karimi et al. [185] developed electroconductive and self-cleaning graphene/ $/ \mathrm{TiO}_{2}$ nanocomposite-coated fabrics. Those fabrics exhibited excellent antimicrobial action toward $S$. aureus and E. coli and antifungal activity toward Candida albicans (C. albicans) with no cytotoxicity. Wang and co-workers [186] prepared a series of $\mathrm{Ag}$ /graphene composites with acrylic acid and $\mathrm{N}, \mathrm{N}^{\prime}$-methylene bisacrylamide with different mass ratios. The Ag/graphene composite hydrogel with an optimal mass ratio at 5:1 (Ag5G1) possessed highest swelling ratio. Most significantly, it could accelerate the healing rate of skin wounds of rats and successfully reconstruct complete and thickened epidermis on the wounds after 15-day treatment. Yang and Lin et al. [187] developed a Ag-based bactericide by anchoring sunlight-driven ultrafine $\mathrm{Ag} / \mathrm{AgCl} \mathrm{NPs}$ on $\mathrm{rGO}(\mathrm{Ag} / \mathrm{AgCl} / \mathrm{rGO})$, which could promote the regeneration of epidermis in burn wound healing. Lu et al. [188] used electrospinning to fabricate chitosan-PVA nanofibers for wound healing.

\subsubsection{Drug delivery}

Graphene is an ideal candidate for drug delivery because of its large surface area, abundant surface chemistry, specific geometry with double side and high-efficiency loading capacity. Dai's group [189] developed pegylated nGO (NGO-PEG) sheets with intrinsic photoluminescence (PL) by conjugating anti-CD20 antibody Rituxan. Chemotherapy drug doxorubicin (DOX) was loaded onto NGOPEG-Rituxan via $\pi$-stacking interaction for in vitro selective killing of cancer cells. Himanshu et al. [190] studied the loading and releasing behavior of bactericidal aminoglycoside antibiotic, gentamicin sulfate from a methanol derived graphene (MDG) sheets. It was found that $\mathrm{MDH}$ possessed a high loading capacity of $2.57 \mathrm{mg} / \mathrm{mg}$, and the release of the drug follows the Korsmeyer-Peppas model. Zhang et al. [191] prepared GO-benzyl penicillin (BP) anion intercalated $\mathrm{Mg}$-Al layered double hydroxide (GO-BP-LDH) hybrid films. The incorporation of BP-LDH and GO could effectively extend the release time for drug delivery, whose kinetics followed the firstorder model. Nafisi et al. [192] presented a systematic study of the adsorption and release process of tetracycline (TC) on GO surface. GO showed a high loading capacity of TC via $\pi-\pi$ and cation- $\pi$ interactions, and the adsorption at equilibrium was observed within only $15 \mathrm{~min}$ following the pseudo-second-order model. Recently, Zhang et al. [193] prepared a cross-linking GO-polyethyleneimine hybrid film containing ciprofloxacin and investigated the release behavior of ciprofloxacin under different $\mathrm{pH}$ values. As a result, it showed slow drug release without an initial burst effect, which was consistent with near zero-order kinetics. Tian et al. [194] developed a GQD-based therapeutic agent with DOX loading and Cy dye conjugation via a cathepsin D-responsive peptide (DOX@GQD$\mathrm{P}-\mathrm{Cy})$ for drug delivery, release and response both in vitro and in vivo. After treatment by DOX@GQD-P-Cy, tumor size was reduced to about $1 / 3$ that that of free DOX. In addition, the agents were used as fluorescent probes for tacking of cell apoptosis induction and real-time evaluation (Fig. 8(b)). Gu et al. developed a cellular protease-mediated graphene-based co-delivery nanosystem incorporating a cell-membrane-targeted anticancer protein and a chemotherapeutic agent for cancer treatment [195]. In further studies, they reported a ATP-responsive anticancer drug delivery system using DNA-graphene nanohybrid aggregates for controlled released of DOX [196]. 

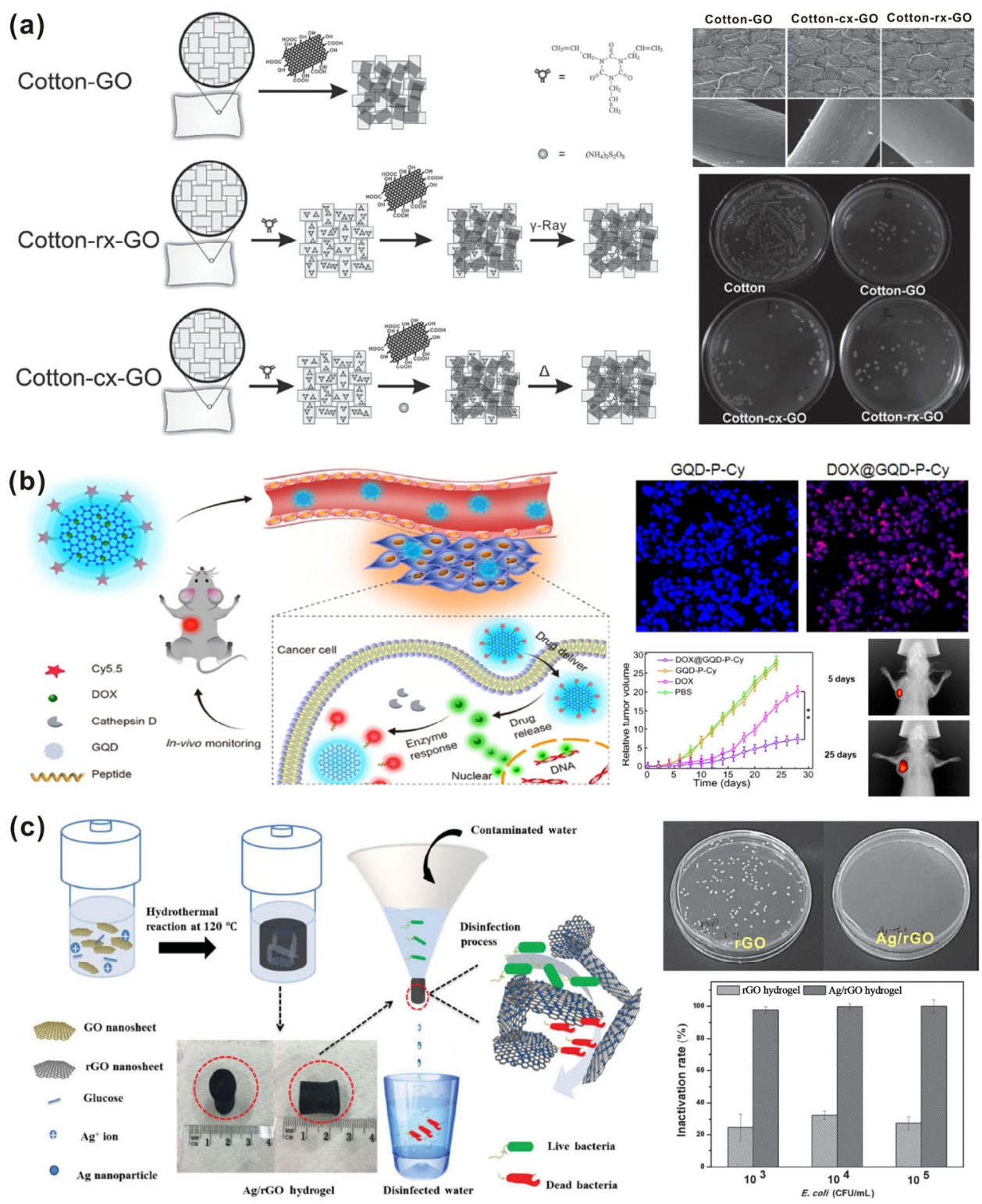

Figure 8 (a) Schematics, SEM image and antibacterial activity for GO modified cotton fabrics. (b) Left: Illustration for GQD-based nanocarriers for drug delivery, release, and response. Right: TUNEL staining of tumor tissue, determination of tumor volumes, and in vivo fluorescent images of mice after treatment. (c) Scheme showing the preparation of $\mathrm{Ag} / \mathrm{rGO}$ hydrogel as bacterial filters for water disinfection, and cell viability of $E$. coli filtered through $\mathrm{rGO}$ and $\mathrm{Ag} / \mathrm{rGO}$ hydrogels. (a) and (c) Adapted with permission from Refs. [184] and [197], ( ) Wiley-VCH Verlag 2013 and 2015. (b) Adapted with permission from Ref. [194], @ American Chemical Society 2017.

\subsubsection{Water disinfection}

Nearly 750 million people around the world have little or zero access to safe drinking water, especially, after natural disasters or in poor urban areas where water disinfection infrastructure is in scarcity. Thus, pathogen-contaminated water leads to millions of deaths every year. Therefore, effective and nontoxic water disinfection nanomaterials is in great demand to kill a broad spectrum of pathogenic microbes. GO-based nanomaterials provide an effective solution for the development of new technologies of water disinfection.

Zhang's group [197] synthesized a 3D Ag/rGO hydrogel through a hydrothermal method, in which the porous $\mathrm{rGO}$ network acted as a support for dispersed AgNPs. When the water flowed through $\mathrm{Ag} / \mathrm{rGO}$ hydrogel, more than $97 \%$ of viable $E$. coli cells were killed owing to membrane damage caused by both AgNPs and rGO nanosheets. For real lake and creek water, the Ag/rGO hydrogel could efficiently inactivate more than $94 \%$ E. coli cells as well as around $99 \%$ of coliforms (Fig. 8(c)), which implies its usage for point-of-use water disinfection application. Recently, the same group co-decorated highly dispersed $\mathrm{TiO}_{2}$ and carbon dots (C-dots) [198] or $\mathrm{WO}_{3}$ nanorods [199] on rGO, and the two ternary nanocomposites showed enhanced photocatalytic activity for inactivation of $E$. coli via electron transfer mechanism. Ray's group [200] developed a PGLa antimicrobial peptide and glutathione conjugated CNT bridged $3 \mathrm{D}$ porous GO membrane, which could capture and inactive E. coli bacteria via synergistic mechanism. Moreover, such membrane has the capability to simultaneously remove E. coli, As(III), As(V), and $\mathrm{Pb}$ (II) from river water sample. Srikanth et al. [201] fabricated $\mathrm{Ag} / \mathrm{Ag}_{2} \mathrm{O}$ core/shell and $\mathrm{Ag} / \mathrm{Ag}_{2} \mathrm{O}$ decorated multi-layered graphene (MLG) nanostructures with excellent adsorption capabilities to remove As(III) from water, as well as high antimicrobial ability against E. coli and S. aureus. Wang et al. [202] reported an activated carbon (AC) electrode coated with graphene oxide-graft-quaternized chitosan (GO-QC) through capacitive deionization disinfection (CDID). It was found that GO-QC/AC CDID electrode could kill at least $99.9999 \%$ of $E$. coli and be easily regenerated in continuous water through the CDID cell, but showed no observable contamination to the water. 


\section{Perspectives and conclusions}

This report summarized various intriguing properties of graphenebased nanomaterials in biosystems. It highlighted the recent research advances in the preparation and exploration of graphene and its derivatives, the extracellular interactions between graphene and biomacromolecules, the cytotoxicity of graphene, in vivo toxicological effects of graphene, as well as their biological applications in antibacterial activities, wound addressing, water purification and drug delivery.

Despite the achievements, there still remains some challenges and difficulties in studies on graphene-based nanomaterials in biosystems. One of the major challenges is understanding the mechanism about the antibacterial activity of graphene and its derivatives. Several studies claimed that GO had little antimicrobial properties; however, most findings gave a controversial view in supporting antibacterial behavior of graphene and its derivatives. In addition, the mechanism and influencing factor of the antibacterial activities of graphene-based nanomaterials have not been fully understood so far. It is still under debate that whether the lateral size, or basal planes, or oxygen content of graphene-based nanomaterials supports its antibacterial activity. Although many findings claimed that graphene-based nanomaterials produced oxidative damage in the antimicrobial activities, unfortunately, it is still questionable whether graphene-based nanomaterials act as direct oxidants or charge transfer mediators, or generate ROS-mediated oxidative stress. Thus, it is worthy of a further investigation to obtain a thorough understanding of the related mechanisms and influencing factors of its antimicrobial activities.

As graphene-based nanomaterials are applied in a wide range of fields, the directly or indirectly cytotoxicity of graphene has drawn great attention about human health and ecosystem risks. Although a great many studies of graphene toxicity have been reported, it is still unclear whether graphene-based nanomaterials affect normal mammalian cells when they kill microorganisms. Due to lack of certain inconsistencies in detailed results and hypotheses of the mechanisms, establishing universal acceptance criteria for toxicity tests is necessary but has not yet been set up. Additionally, a better understanding of the mechanism of graphene toxicology needs further elaboration in future, which might circumvent the problems and find appropriate ways to realize the biocompatibility of graphene.

\section{Acknowledgements}

This work was financially supported by the National Key Research and Development Program (No. 2016YFA0201200), the Shanghai Municipal Natural Science Foundation (No. 17ZR1412100), the Key Laboratory of Interfacial Physics and Technology, the Chinese Academy of Sciences (No. CASKL-IPT1603), the Talent Program of Shanghai University of Engineering Science, the Startup Foundation for Doctors of Shanghai University of Engineering Science, and the National Natural Science Foundation of China (Nos. 81870749, 21373260, 31470960 and 51375294).

\section{References}

[1] Novoselov, K. S.; Geim, A. K.; Morozov, S. V.; Jiang, D.; Zhang, Y.; Dubonos, S. V.; Grigorieva, I. V.; Firsov, A. A. Electric field effect in atomically thin carbon films. Science 2004, 306, 666-669.

[2] Huang, X.; Yin, Z. Y.; Wu, S. X.; Qi, X. Y.; He, Q. Y.; Zhang, Q. C.; Yan, Q. Y.; Boey, F.; Zhang, H. Graphene-based materials: Synthesis, characterization, properties, and applications. Small 2011, 7, 1876-1902.

[3] Hu, X. G.; Zhou, Q. X. Health and ecosystem risks of graphene. Chem. Rev. 2013, 113, 3815-3835.

[4] Tang, L.H.; Wang, Y.; Li, J. H. The graphene/nucleic acid nanobiointerface. Chem. Soc. Rev. 2015, 44, 6954-6580.

[5] Zheng, H. Z.; Ma, R. L.; Gao, M.; Tian, X.; Li, Y. Q.; Zeng, L. W.; Li, R.
B. Antibacterial applications of graphene oxides: Structure-activity relationships, molecular initiating events and biosafety. Sci. Bull. 2018, 63, 133-142.

[6] Park, S.; Ruoff, R. S. Chemical methods for the production of graphenes. Nat. Nanotechnol. 2009, 4, 217-224.

[7] Ponomarenko, L. A.; Schedin, F.; Katsnelson, M. I.; Yang, R.; Hill, E. W.; Novoselov, K. S.; Geim, A. K. Chaotic dirac billiard in graphene quantum dots. Science 2008, 320, 356-358.

[8] Slota, M.; Keerthi, A.; Myers, W. K.; Tretyakov, E.; Baumgarten, M.; Ardavan, A.; Sadeghi, H.; Lambert, C. J.; Narita, A.; Müllen, K. et al. Magnetic edge states and coherent manipulation of graphene nanoribbons. Nature 2018, 557, 691-695.

[9] Rozpłoch, F.; Patyk, J.; Stankowski, J. Graphenes bonding forces in graphite. Acta Phys. Pol. A 2007, 112, 557-562.

[10] Hernandez, Y.; Nicolosi, V.; Lotya, M.; Blighe, F. M.; Sun, Z. Y.; De, S.; McGovern, I. T.; Holland, B.; Byrne, M.; Gun'Ko, Y. K. et al. High-yield production of graphene by liquid-phase exfoliation of graphite. Nat. Nanotechnol. 2008, 3, 563-568.

[11] Kim, K. S.; Zhao, Y.; Jang, H.; Lee, S. Y.; Kim, J. M.; Kim, K. S.; Ahn, J. H.; Kim, P.; Choi, J. Y.; Hong, B. H. Large-scale pattern growth of graphene films for stretchable transparent electrodes. Nature 2009, 457, 706-710.

[12] Bae, S.; Kim, H.; Lee, Y.; Xu, X. F.; Park, J. S.; Zheng, Y.; Balakrishnan, J.; Lei, T.; Kim, H. R.; Song, Y. I. et al. Roll-to-roll production of 30-inch graphene films for transparent electrodes. Nat. Nanotechnol. 2010, 5, 574-578.

[13] Chen, C. Y.; Avila, J.; Arezki, H.; Nguyen, V. L.; Shen, J. H.; MuchaKruczyński, M.; Yao, F.; Boutchich, M.; Chen, Y.; Lee, Y. H. et al. Large local lattice expansion in graphene adlayers grown on copper. Nat. Mater. 2018, 17, 450-455.

[14] Berger, C.; Song, Z. M.; Li, X. B.; Wu, X. S.; Brown, N.; Naud, C.; Mayou, D.; Li, T. B.; Hass, J.; Marchenkov, A. N. et al. Electronic confinement and coherence in patterned epitaxial graphene. Science 2006, 312, 1191-1196.

[15] Zhang, L. C.; Shi, Z. W.; Liu, D. H.; Yang, R.; Shi, D. X.; Zhang, G. Y. Vapour-phase graphene epitaxy at low temperatures. Nano Res. 2012, 5 , 258-264.

[16] Shao, Y.; Liu, Z. L.; Cheng, C.; Wu, X.; Liu, H.; Liu, C.; Wang, J. O.; Zhu, S. Y.; Wang, Y. Q.; Shi, D. X. et al. Epitaxial growth of flat antimonene monolayer: A new honeycomb analogue of graphene. Nano Lett. 2018, 18, 2133-2139.

[17] Hummers, W. S. H. Jr.; Offeman, R. E. Preparation of graphitic oxide. J. Am. Chem. Soc. 1958, 80, 1339-1339.

[18] Dikin, D. A.; Stankovich, S.; Zimney, E. J.; Piner, R. D.; Dommett, G. H. B.; Evmenenko, G.; Nguyen, S. T.; Ruoff, R. S. Preparation and characterization of graphene oxide paper. Nature 2007, 448, 457-460.

[19] Marcano, D. C.; Kosynkin, D. V.; Berlin, J. M.; Sinitskii, A.; Sun, Z. Z.; Slesarev, A.; Alemany, L. B.; Lu, W.; Tour, J. M. Improved synthesis of graphene oxide. ACS Nano 2010, 4, 4806-4814.

[20] Li, D.; Müller, M. B.; Gilje, S.; Kaner, R. B.; Wallace, G. G. Processable aqueous dispersions of graphene nanosheets. Nat. Nanotechnol. 2008, 3 , $101-105$

[21] Voiry, D.; Yang, J.; Kupferberg, J.; Fullon, R.; Lee, C.; Jeong, H. Y.; Shin, H. S.; Chhowalla, M. High-quality graphene via microwave reduction of solution-exfoliated graphene oxide. Science 2016, 353, 1413-1416.

[22] Nam, J. M.; Thaxton, C. S.; Mirkin, C. A. Nanoparticle-based bio-bar codes for the ultrasensitive detection of proteins. Science 2003, 301, 1884-1886.

[23] Wang, P. J.; Wan, Y.; Ali, A.; Deng, S. Y.; Su, Y.; Fan, C. H.; Yang, S. L. Aptamer-wrapped gold nanoparticles for the colorimetric detection of omethoate. Sci. China Chem. 2016, 59, 237-242.

[24] Katz, E.; Willner, I. Integrated nanoparticle-biomolecule hybrid systems: Synthesis, properties, and applications. Angew. Chem., Int. Ed. 2004, 43, 6042-6108.

[25] Xu, Y.; Zhao, Y.; Zhang, Y. J.; Cui, Z. F.; Wang, L. H.; Fan, C. H.; Gao, J. M.; Sun, Y. H. Angiopep-2-conjugated $\mathrm{Ag}_{2} \mathrm{~S}$ quantum dot for NIR-II imaging of brain tumors. Acta Chim. Sin. 2018, 76, 393-399.

[26] Cui, X. H.; Chen, H. Y.; Yang, T. Research progress on the preparation and application of nano-sized molybdenum disulfide. Acta Chim. Sin. 2016, 74, $392-400$.

[27] Lu, N.; Gao, A. R.; Dai, P. F.; Song, S. P.; Fan, C. H.; Wang, Y. L.; Li, T. CMOS-compatible silicon nanowire field-effect transistors for ultrasensitive and label-free microRNAs sensing. Small 2014, 10, 2022-2028. 
[28] Ramanathan, T.; Abdala, A. A.; Stankovich, S.; Dikin, D. A.; HerreraAlonso, M.; Piner, R. D.; Adamson, D. H.; Schniepp, H. C.; Chen, X.; Ruoff, R. S. et al. Functionalized graphene sheets for polymer nanocomposites. Nat. Nanotechnol. 2008, 3, 327-331.

[29] Stuart, M. A. C.; Huck, W. T. S.; Genzer, J.; Müller, M.; Ober, C.; Stamm, M.; Sukhorukov, G. B.; Szleifer, I.; Tsukruk, V. V.; Urban, M. et al. Emerging applications of stimuli-responsive polymer materials. Nat. Mater. 2010, 9, 101-113.

[30] Huang, X.; Qi, X. Y.; Boey, F.; Zhang, H. Graphene-based composites. Chem. Soc. Rev. 2012, 41, 666-686.

[31] Muszynski, R.; Seger, B.; Kamat, P. V. Decorating graphene sheets with gold nanoparticles. J. Phys. Chem. C 2008, 112, 5263-5266.

[32] Cao, A. N.; Liu, Z.; Chu, S. S.; Wu, M. H.; Ye, Z. M.; Cai, Z. W.; Chang, Y. L.; Wang, S. F.; Gong, Q. H.; Liu, Y. F. A facile one-step method to produce graphene-CdS quantum dot nanocomposites as promising optoelectronic materials. Adv. Mater. 2010, 22, 103-106.

[33] Ji, H. W.; Sun, H. J.; Qu, X. G. Antibacterial applications of graphenebased nanomaterials: Recent achievements and challenges. Adv. Drug Deliver. Rev. 2016, 105, 176-189.

[34] Liu, J. W.; Cao, Z. H.; Lu, Y. Functional nucleic acid sensors. Chem. Rev. 2009, 109, 1948-1998.

[35] Ge, Z. L.; Pei, H.; Wang, L. H.; Song, S. P.; Fan, C. H. Electrochemical single nucleotide polymorphisms genotyping on surface immobilized three-dimensional branched DNA nanostructure. Sci. China Chem. 2011, 54,1273 .

[36] Seeman, N. C. DNA in a material world. Nature 2003, 421, 427-431.

[37] Zhang, H. Z.; Zhang, Z. Q.; Wang, F.; Zhou, T.; Wang, X. F.; Zhang, G. D.; Liu, T. T.; Liu, S. Z. Application of structural DNA nanotechnology. Acta Phys. Chim. Sin. 2017, 33, 1520-1532.

[38] Pinheiro, A. V.; Han, D. R.; Shih, W. M.; Yan, H. Challenges and opportunities for structural DNA nanotechnology. Nat. Nanotechnol. 2011, 6, 763-772.

[39] Ye, D. K.; Zuo, X. L.; Fan, C. H. DNA nanostructure-based engineering of the biosensing interface for biomolecular detection. Prog. Chem. 2017, 29, $36-46$.

[40] Liu, D. G.; Park, S. H.; Reif, J. H.; LaBean, T. H. DNA nanotubes selfassembled from triple-crossover tiles as templates for conductive nanowires. Proc. Natl. Acad. Sci. USA 2004, 101, 717-722.

[41] Lin, C. X.; Katilius, E.; Liu, Y.; Zhang, J. P.; Yan, H. Self-assembled signaling aptamer DNA arrays for protein detection. Angew. Chem., Int. Ed. 2006, 45, 5296-5301.

[42] Rothemund, P. W. K. Folding DNA to create nanoscale shapes and patterns. Nature 2006, 440, 297-302.

[43] Chao, J.; Zhang, Y. N.; Zhu, D.; Liu, B.; Cui, C. J.; Su, S.; Fan, C. H.; Wang, L. H. Hetero-assembly of gold nanoparticles on a DNA origami template. Sci. China Chem. 2016, 59, 730-734.

[44] Han, D. R.; Pal, S.; Nangreave, J.; Deng, Z. T.; Liu, Y.; Yan, H. DNA origami with complex curvatures in three-dimensional space. Science 2011, 332, 342-346.

[45] Fan, C. H.; Fang, X. H. Special topic for "single-molecule, single-particle and single-cell bioimaging". Sci. China Chem. 2017, 60, 1265-1266.

[46] Gao, Z. S.; Deng, S. H.; Li, J.; Wang, K.; Li, J. J.; Wang, L. H.; Fan, C. H. Sub-diffraction-limit cell imaging using a super-resolution microscope with simplified pulse synchronization. Sci. China Chem. 2017, 60, 1305-1309.

[47] Wang, S. P.; Deng, S. H.; Cai, X. Q.; Hou, S. G.; Li, J. J.; Gao, Z. S.; Li, J.; Wang, L. H.; Fan, C. H. Superresolution imaging of telomeres with continuous wave stimulated emission depletion (STED) microscope. Sci. China Chem. 2016, 59, 1519-1524.

[48] Liu, Q.; Sun, Y.; Yang, T. S.; Feng, W.; Li, C. G.; Li, F. Y. Sub-10 nm hexagonal lanthanide-doped $\mathrm{NaLuF}_{4}$ upconversion nanocrystals for sensitive bioimaging in vivo. J. Am. Chem. Soc. 2016, 133, 17122-17125.

[49] Yang, K.; Feng, L. Z.; Shi, X. Z.; Liu, Z. Nano-graphene in biomedicine: Theranostic applications. Chem. Soc. Rev. 2013, 42, 530-547.

[50] Dreaden, E. C.; Alkilany, A. M.; Huang, X. H.; Murphy, C. J.; El-Sayed, M. A. The golden age: Gold nanoparticles for biomedicine. Chem. Soc. Rev. 2012, 41, 2740-2779.

[51] Su, Y. Y.; Peng, T. H.; Xing, F. F.; Li, D.; Fan, C. H. Nanoplasmonic biological sensing and imaging. Acta Chim. Sin. 2017, 75, 1036-1046.

[52] Long, Y. T.; Fan, C. H. Nanosensors. Acta Chim. Sin. 2017, 75, 1021-1022.

[53] Chao, J.; Fan, C. H. A photoelectrochemical sensing strategy for biomolecular detection. Sci. China Chem. 2015, 58, 834.

[54] Patil, A. J.; Vickery, J. L.; Scott, T. B.; Mann, S. Aqueous stabilization and self-assembly of graphene sheets into layered bio-nanocomposites using DNA. Adv. Mater. 2009, 21, 3159-3164.

[55] Lu, C. H.; Yang, H. H.; Zhu, C. L.; Chen, X.; Chen, G. N. A graphene platform for sensing biomolecules. Angew. Chem., Int. Ed. 2009, 48, 4785-4787.

[56] He, S. J.; Song, B.; Li, D.; Zhu, C. F.; Qi, W. P.; Wen, Y. Q.; Wang, L. H.; Song, S. P.; Fang, H. P.; Fan, C. A graphene nanoprobe for rapid, sensitive, and multicolor fluorescent DNA analysis. Adv. Funct. Mater. 2010, 20, 453-459.

[57] Chang, H. X.; Tang, L. H.; Wang, Y.; Jiang, J. H.; Li, J. H. Graphene fluorescence resonance energy transfer aptasensor for the thrombin detection. Anal. Chem. 2010, 82, 2341-2346.

[58] Wu, M.; Kempaiah, R.; Huang, P. J. J.; Maheshwari, V.; Liu, J. W. Adsorption and desorption of DNA on graphene oxide studied by fluorescently labeled oligonucleotides. Langmuir 2011, 27, 2731-2738.

[59] Kotikam, V.; Fernandes, M.; Kumar, V. A. Comparing the interactions of DNA, polyamide (PNA) and polycarbamate nucleic acid (PCNA) oligomers with graphene oxide (GO). Phys. Chem. Chem. Phys. 2012, 14, 15003-15006.

[60] Cui, L.; Chen, Z. R.; Zhu, Z.; Lin, X. Y.; Chen, X.; Yang, C. J. Stabilization of ssRNA on graphene oxide surface: An effective way to design highly robust RNA probes. Anal. Chem. 2013, 85, 2269-2275.

[61] Zhang, H.; Jia, S. S.; Lv, M.; Shi, J. Y.; Zuo, X. L.; Su, S.; Wang, L. H.; Huang, W.; Fan, C. H.; Huang, Q. Size-dependent programming of the dynamic range of graphene oxide-DNA interaction-based ion sensors. Anal. Chem. 2014, 86, 4047-4051.

[62] Xu, S. C.; Zhan, J.; Man, B. Y.; Jiang, S. Z.; Yue, W. W.; Gao, S. B.; Guo, C. G.; Liu, H. P.; Li, Z. H.; Wang, J. H. et al. Real-time reliable determination of binding kinetics of DNA hybridization using a multi-channel graphene biosensor. Nat. Commun. 2017, 8, 14902.

[63] Heerema, S. J.; Vicarelli, L.; Pud, S.; Schouten, R. N.; Zandbergen, H. W.; Dekker, C. Probing DNA translocations with inplane current signals in a graphene nanoribbon with a nanopore. ACS Nano 2018, 12, 2623-2633.

[64] Pei, H.; Li, J.; Lv, M.; Wang, J. Y.; Gao, J. M.; Lu, J. X.; Li, Y. P.; Huang, Q.; Hu, J.; Fan, C. H. A graphene-based sensor array for high-precision and adaptive target identification with ensemble aptamers. J. Am. Chem. Soc. 2012, 134, 13843-13849.

[65] Feng, L. Y.; Li, W.; Ren, J. S.; Qu, X. G. Electrochemically and DNAtriggered cell release from ferrocene/ $\beta$-cyclodextrin and aptamer modified dualfunctionalized graphene substrate. Nano Res. 2015, 8, 887-899.

[66] Liu, M.; Song, J. P.; Shuang, S. M.; Dong, C.; Brennan, J. D.; Li, Y. F. A graphene-based biosensing platform based on the release of DNA probes and rolling circle amplification. ACS Nano 2014, 8, 5564-5573.

[67] Liu, S. Z.; Zhang, Z. Q.; Wang, F.; Zhou, T.; Wang, X. F.; Zhang, G. D.; Liu, T. T.; Zhang, H. Z. Study on the synthesis of DNA via rolling circle amplification. Acta Phys. Chim. Sin 2017, 33, 2052-2057.

[68] Li, F.; Liu, X. G.; Zhao, B.; Yan, J.; Li, Q.; Aldalbahi, A.; Shi, J. Y.; Song, S. P.; Fan, C. H.; Wang, L. H. Graphene nanoprobes for real-time monitoring of isothermal nucleic acid amplification. ACS Appl. Mater. Interfaces 2017, 9, 15245-15253.

[69] Liu, X. Q.; Aizen, R.; Freeman, R.; Yehezkeli, O.; Willner, I. Multiplexed aptasensors and amplified DNA sensors using functionalized graphene oxide: Application for logic gate operations. ACS Nano 2012, 6, 3553-3563.

[70] Tian, Y.; Wang, Y.; Xu, Y.; Liu, Y.; Li, D.; Fan, C. H. A highly sensitive chemiluminescence sensor for detecting mercury (II) ions: A combination of Exonuclease III-aided signal amplification and graphene oxide-assisted background reduction. Sci. China Chem 2015, 58, 514-518.

[71] Gowtham, S.; Scheicher, R. H.; Ahuja, R.; Pandey, R.; Karna, S. P. Physisorption of nucleobases on graphene: Density-functional calculations. Phys. Rev. B 2007, 76, 033401.

[72] Varghese, N.; Mogera, U.; Govindaraj, A.; Das, A.; Maiti, P. K.; Sood, A. K.; Rao, C. N. R. Binding of DNA nucleobases and nucleosides with graphene. Chemphyschem 2009, 10, 206-210.

[73] Panigrahi, S.; Bhattacharya, A.; Banerjee, S.; Bhattacharyya, D. Interaction of nucleobases with wrinkled graphene surface: Dispersion corrected DFT and AFM studies. J. Phys. Chem. C 2012, 116, 4374-4379.

[74] Zhang, Y. Y.; Li, M.; Li, Z. H.; Li, Q.; Aldalbahi, A.; Shi, J. Y.; Wang, L. H.; Fan, C. H.; Zuo, X. L. Recognizing single phospholipid vesicle collisions 
on carbon fiber nanoelectrode. Sci. China Chem. 2017, 60, 1474-1480.

[75] Duan, X. X.; Li, Y.; Rajan, N. K.; Routenberg, D. A.; Modis, Y.; Reed, M. A. Quantification of the affinities and kinetics of protein interactions using silicon nanowire biosensors. Nat. Nanotechnol. 2012, 7, 401-407.

[76] Li, J.; Kuang, Y.; Shi, J. F.; Zhou, J.; Medina, J. E.; Zhou, R.; Yuan, D.; Yang, C. H.; Wang, H. M.; Yang, Z. M. et al. Enzyme-instructed intracellular molecular self-assembly to boost activity of cisplatin against drug-resistant ovarian cancer cells. Angew. Chem., Int. Ed. 2015, 127, 13505-13509.

[77] Jung, J. H.; Cheon, D. S.; Liu, F.; Lee, K. B.; Seo, T. S. A graphene oxide based immuno-biosensor for pathogen detection. Angew. Chem., Int. Ed. Int. Ed. 2010, 49, 5708-5711.

[78] Wang, L. H.; Pu, K. Y.; Li, J.; Qi, X. Y.; Li, H.; Zhang, H.; Fan, C. H.; Liu, B. A graphene-conjugated oligomer hybrid probe for light-up sensing of lectin and Escherichia coli. Adv. Mater. 2011, 23, 4386-4391.

[79] Chou, S. S.; De, M.; Luo, J. Y.; Rotello, V. M.; Huang, J. X.; Dravid, V. P. Nanoscale graphene oxide (nGO) as artificial receptors: Implications for biomolecular interactions and sensing. J. Am. Chem. Soc. 2012, 134, $16725-16733$.

[80] Tan, X. F.; Feng, L. Z.; Zhang, J.; Yang, K.; Zhang, S.; Liu, Z.; Peng, R. Functionalization of graphene oxide generates a unique interface for selective serum protein interactions. ACS Appl. Mater. Interaces 2013, 5 , 1370-1377.

[81] Gan, S. Y.; Zhong, L. J.; Han, D. X.; Niu, L.; Chi, Q. J. Probing bio-nano interactions between blood proteins and monolayer-stabilized graphene sheets. Small 2015, 11, 5814-5825.

[82] Feng, B. Y.; Guo, L. J.; Wang, L. H.; Li, F.; Lu, J. X.; Gao, J. M.; Fan, C. H.; Huang, Q. A graphene oxide-based fluorescent biosensor for the analysis of peptide-receptor interactions and imaging in somatostatin receptor subtype 2 overexpressed tumor cells. Anal. Chem. 2013, 85, 7732-7737.

[83] Zou, X. Q.; Wei, S.; Jasensky, J.; Xiao, M. Y.; Wang, Q. M.; Brooks, C. L.; Chen, Z. Molecular interactions between graphene and biological molecules. J. Am. Chem. Soc. 2017, 139, 1928-1936.

[84] Chen, Y. J.; Chen, Z. H.; Sun, Y. X.; Lei, J. T.; Wei, G. H. Mechanistic insights into the inhibition and size effects of graphene oxide nanosheets on the aggregation of an amyloid- $\beta$ peptide fragment. Nanoscale 2018, 10, 8989-8997.

[85] Jang, H.; Ryoo, S. R.; Kim, Y. K.; Yoon, S.; Kim, H.; Han, S. W.; Choi, B. S.; Kim, D. E.; Min, D. H. Discovery of hepatitis C virus NS3 helicase inhibitors by a multiplexed, high-throughput helicase activity assay based on graphene oxide. Angew. Chem., Int. Ed. 2013, 52, 2340-2344.

[86] Song, Z. Y.; Wang, X. Y.; Zhu, G. X.; Nian, Q. G.; Zhou, H. Y.; Yang, D.; Qin, C. F.; Tang, R. K. Virus capture and destruction by label-free graphene oxide for detection and disinfection applications. Small 2015, 11, 1171-1176.

[87] Deokar, A. R.; Nagvenkar, A. P.; Kalt, I.; Shani, L.; Yeshurun, Y.; Gedanken, A.; Sarid, R. Graphene-based "hot plate" for the capture and destruction of the herpes simplex virus type 1. Bioconjugate Chem. 2017, 28, $1115-1122$.

[88] Sametband, M.; Kalt, I.; Gedanken, A.; Sarid, R. Herpes simplex virus type-1 attachment inhibition by functionalized graphene oxide. ACS Appl. Mater. Interfaces 2014, 6, 1228-1235.

[89] Gholami, M. F.; Lauster, D.; Ludwig, K.; Storm, J.; Ziem, B.; Severin, N.; Böttcher, C.; Rabe, J. P.; Herrmann, A.; Adeli, M. et al. Functionalized graphene as extracellular matrix mimics: Toward well-defined 2D nanomaterials for multivalent virus interactions. Adv. Funct. Mater. 2017, 27, 1606477.

[90] Zhan, L.; Li, C. M.; Wu, W. B.; Huang, C. Z. A colorimetric immunoassay for respiratory syncytial virus detection based on gold nanoparticlesgraphene oxide hybrids with mercury-enhanced peroxidase-like activity. Chem. Commun. 2014, 50, 11526-11528.

[91] Afsahi, S.; Lerner, M. B.; Goldstein, J. M.; Lee, J.; Tang, X. L.; Bagarozzi, D. A. Jr.; Pan, D.; Locascio, L.; Walker, A.; Barron, F. et al. Novel graphene-based biosensor for early detection of Zika virus infection. Biosens. Bioelectron. 2018, 100, 85-88.

[92] Lee, Y. M.; Jung, B.; Kim, Y. H.; Park, A. R.; Han, S.; Choe, W. S.; Yoo, P. $\mathrm{J}$. Nanomesh-structured ultrathin membranes harnessing the unidirectional alignment of viruses on a graphene-oxide film. Adv. Mater. 2014, 26, 3899-3904.

[93] Chen, H. Q.; Müller, M. B.; Gilmore, K. J.; Wallace, G. G.; Li, D. Mechanically strong, electrically conductive, and biocompatible graphene paper. $A d v$.
Mater. 2008, 20, 3557-3561.

[94] Lee, W. C.; Lim, C. H. Y. X.; Shi, H.; Tang, L. A. L.; Wang, Y.; Lim, C. T.; Loh, K. P. Origin of enhanced stem cell growth and differentiation on graphene and graphene oxide. ACS Nano 2011, 5, 7334-7341.

[95] Rastogi, S. K.; Raghavan, G.; Yang, G.; Cohen-Karni, T. Effect of graphene on nonneuronal and neuronal cell viability and stress. Nano Lett. 2017, 17, 3297-3301.

[96] Guo, C. X.; Zheng, X. T.; Lu, Z. S.; Lou, X. W.; Li, C. M. Biointerface by cell growth on layered graphene-artificial peroxidase-protein nanostructure for in situ quantitative molecular detection. Adv. Mater. 2010, 22, 5164-5167.

[97] Bardhan, N. M.; Kumar, P. V.; Li, Z. Y.; Ploegh, H. L.; Grossman, J. C.; Belcher, A. M.; Chen, G. Y. Enhanced cell capture on functionalized graphene oxide nanosheets through oxygen clustering. ACS Nano 2017, 11, $1548-1558$.

[98] Shah, S.; Yin, P. T.; Uehara, T. M.; Chueng, S. T. D.; Yang, L. T.; Lee, K. B. Guiding stem cell differentiation into oligodendrocytes using graphenenanofiber hybrid scaffolds. Adv. Mater. 2014, 26, 3673-3680.

[99] Li, W.; Wang, J. S.; Ren, J. S.; Qu, X. G. 3D graphene oxide-polymer hydrogel: Near-infrared light-triggered active scaffold for reversible cell capture and on-demand release. Adv. Mater. 2013, 25, 6737-6743.

[100] Lu, J. Y.; Zhang, X. X.; Zhu, Q. Y.; Zhang, F. R.; Huang, W. T.; Ding, X. Z.; Xia, L. Q.; Luo, H. Q.; Li, N. B. Highly tunable and scalable fabrication of $3 \mathrm{D}$ flexible graphene micropatterns for directing cell alignment. ACS Appl. Mater. Interfaces 2018, 10, 17704-17713.

[101] Chang, Y. L.; Yang, S. T.; Liu, J. H.; Dong, E. Y.; Wang, Y. W.; Cao, A. N.; Liu, Y. F.; Wang, H. F. In vitro toxicity evaluation of graphene oxide on A549 cells. Toxicol Lett 2011, 200, 201-210.

[102] Chong, Y.; Ma, Y. F.; Shen, H.; Tu, X. L.; Zhou, X.; Xu, J. Y.; Dai, J. W.; Fan, S. J.; Zhang, Z. J. The in vitro and in vivo toxicity of graphene quantum dots. Biomaterials 2014, 35, 5041-5048.

[103] Zhang, Y. B.; Ali, S. F.; Dervishi, E.; Xu, Y.; Li, Z. R.; Casciano, D.; Biris, A. S. Cytotoxicity effects of graphene and single-wall carbon nanotubes in neural phaeochromocytoma-derived PC12 cells. ACS Nano 2010, 4, 3181-3186.

[104] Vallabani, N. V. S.; Mittal, S.; Shukla, R. K.; Pandey, A. K.; Dhakate, S. R.; Pasricha, R.; Dhawan, A. Toxicity of graphene in normal human lung cells (BEAS-2B). J. Biomed. Nanotechnol. 2011, 7, 106-107.

[105] Hu, W. B.; Peng, C.; Lv, M.; Li, X. M.; Zhang, Y. J.; Chen, N.; Fan, C. H.; Huang, Q. Protein corona-mediated mitigation of cytotoxicity of graphene oxide. ACS Nano 2011, 5, 3693-3700.

[106] Chong, Y.; Ge, C. C.; Yang, Z. X.; Garate, J. A.; Gu, Z. L.; Weber, J. K.; Liu, J. J.; Zhou, R. H. Reduced cytotoxicity of graphene nanosheets mediated by blood-protein coating. ACS Nano 2015, 9, 5713-5724.

[107] Zhang, H.; Cheng, P.; Yang, J. Z.; Lv, M.; Liu, R.; He, D. N.; Fan, C. H.; Huang, Q. Uniform ultrasmall graphene oxide nanosheets with low cytotoxicity and high cellular uptake. ACS Appl. Mater. Interfaces 2013, 5, 1761-1767.

[108] Mittal, S.; Kumar, V.; Dhiman, N.; Chauhan, L. K. S.; Pasricha, R.; Pandey, A. K. Physico-chemical properties based differential toxicity of graphene oxide/reduced graphene oxide in human lung cells mediated through oxidative stress. Sci. Rep. 2016, 6, 39548.

[109] Vranic, S.; Rodrigues, A. F.; Buggio, M.; Newman, L.; White, M. R. H.; Spiller, D. G.; Bussy, C.; Kostarelos, K. Live imaging of label-free graphene oxide reveals critical factors causing oxidative-stress-mediated cellular responses. ACS Nano 2018, 12, 1373-1389.

[110] Li, R. B.; Guiney, L. M.; Chang, C. H.; Mansukhani, N. D.; Ji, Z. X.; Wang, X.; Liao, Y. P.; Jiang, W.; Sun, B. B.; Hersam, M. C. et al. Surface oxidation of graphene oxide determines membrane damage, lipid peroxidation, and cytotoxicity in macrophages in a pulmonary toxicity model. ACS Nano 2018, 12, 1390-1402.

[111] Li, Y.; Liu, Y.; Fu, Y. J.; Wei, T. T.; Le Guyader, L.; Gao, G.; Liu, R. S.; Chang, Y. Z.; Chen, C. Y. The triggering of apoptosis in macrophages by pristine graphene through the MAPK and TGF-beta signaling pathways. Biomaterials 2012, 33, 402-411.

[112] Chen, G. Y.; Yang, H. J.; Lu, C. H.; Chao, Y. C.; Hwang, S. M.; Chen, C. L.; Lo, K. W.; Sung, L. Y.; Luo, W. Y.; Tuan, H. Y. et al. Simultaneous induction of autophagy and toll-like receptor signaling pathways by graphene oxide. Biomaterials 2012, 33, 6559-6569.

[113] Wang, L.; Li, X. M.; Han, Y. P.; Wang, T.; Zhao, Y.; Ali, A.; El-Sayed, N. 
N.; Shi, J. Y.; Wang, W. F.; Fan, C. H. et al. Quantum dots protect against $\mathrm{MPP}^{+}$-induced neurotoxicity in a cell model of Parkinson's disease through autophagy induction. Sci. China Chem. 2016, 59, 1486-1491.

[114] Pieper, H.; Chercheja, S.; Eigler, S.; Halbig, C. E.; Filipovic, M. R.; Mokhir, A. Endoperoxides revealed as origin of the toxicity of graphene oxide. Angew. Chem., Int. Ed. 2016, 55, 405-407.

[115] Zhu, J. Q.; Xu, M.; Gao, M.; Zhang, Z. H.; Xu, Y.; Xia, T.; Liu, S. J. Graphene oxide-induced perturbation to plasma membrane and cytoskeletal meshwork sensitize cancer cells to chemotherapeutic agents. ACS Nano 2017, 11, 2637-2651.

[116] Zhang, W. D.; Wang, C.; Li, Z. J.; Lu, Z. Z.; Li, Y. Y.; Yin, J. J.; Zhou, Y. T.; Gao, X. F.; Fang, Y.; Nie, G. et al. Unraveling stress-induced toxicity properties of graphene oxide and the underlying mechanism. Adv. Mater. 2012, 24, 5391-5397.

[117] Zhang, X. Y.; Yin, J. L.; Peng, C.; Hu, W. Q.; Zhu, Z. Y.; Li, W. X.; Fan, C. H.; Huang, Q. Distribution and biocompatibility studies of graphene oxide in mice after intravenous administration. Carbon 2011, 49, 986-995.

[118] Yang, K.; Wan, J. M.; Zhang, S.; Zhang, Y. J.; Lee, S. T.; Liu, Z. In vivo pharmacokinetics, long-term biodistribution, and toxicology of PEGylated graphene in mice. ACS Nano 2011, 5, 516-522.

[119] Li, B.; Zhang, X. Y.; Yang, J. Z.; Zhang, Y. J.; Li, W. X.; Fan, C. H.; Huang, Q. Influence of polyethylene glycol coating on biodistribution and toxicity of nanoscale graphene oxide in mice after intravenous injection. Int. J. Nanomedicine 2014, 9, 4697-4707.

[120] Zhang, S.; Yang, K.; Feng, L. Z.; Liu, Z. In vitro and in vivo behaviors of dextran functionalized graphene. Carbon 2011, 49, 4040-4049.

[121] Yang, K.; Gong, H.; Shi, X. Z.; Wan, J. M.; Zhang, Y. J.; Liu, Z. In vivo biodistribution and toxicology of functionalized nano-graphene oxide in mice after oral and intraperitoneal administration. Biomaterials 2013, 34, 2787-2795.

[122] Syama, S.; Paul, W.; Sabareeswaran, A.; Mohanan, P. V. Raman spectroscopy for the detection of organ distribution and clearance of PEGylated reduced graphene oxide and biological consequences. Biomaterials 2017, 131, 121-130.

[123] Nurunnabi, M.; Khatun, Z.; Huh, K. M.; Park, S. Y.; Lee, D. Y.; Cho, K. J.; Lee, Y. K. In vivo biodistribution and toxicology of carboxylated graphene quantum dots. ACS Nano 2013, 7, 6858-6867.

[124] Ema, M.; Gamo, M.; Honda, K. A review of toxicity studies on graphenebased nanomaterials in laboratory animals. Regul. Toxicol. Pharmacol. 2017, 85, 7-24.

[125] Gollavelli, G.; Ling, Y. C. Multi-functional graphene as an in vitro and in vivo imaging probe. Biomaterials 2012, 33, 2532-2545.

[126] Akhavan, O.; Ghaderi, E.; Rahimi, K. Adverse effects of graphene incorporated in $\mathrm{TiO}_{2}$ photocatalyst on minuscule animals under solar light irradiation. J. Mater. Chem. 2012, 22, 23260-23266.

[127] Xu, S.; Zhang, Z. Y.; Chu, M. Q. Long-term toxicity of reduced graphene oxide nanosheets: Effects on female mouse reproductive ability and offspring development. Biomaterials 2015, 54, 188-200.

[128] Ma, J.; Liu, R.; Wang, X.; Liu, Q.; Chen, Y.; Valle, R. P.; Zuo, Y. Y.; Xia, T.; Liu, S. J. Crucial role of lateral size for graphene oxide in activating macrophages and stimulating pro-inflammatory responses in cells and animals. ACS Nano 2015, 9, 10498-10515.

[129] Begum, P.; Ikhtiari, R.; Fugetsu, B. Graphene phytotoxicity in the seedling stage of cabbage, tomato, red spinach, and lettuce. Carbon 2011, 49, 3907-3919.

[130] Zhang, P.; Zhang, R. R.; Fang, X. Z.; Song, T. Q.; Cai, X. D.; Liu, H. J.; $\mathrm{Du}, \mathrm{S}$. T. Toxic effects of graphene on the growth and nutritional levels of wheat (Triticum aestivum L.): Short- and long-term exposure studies. J. Hazard. Mater. 2016, 317, 543-551.

[131] Wang, Q. Q.; Zhao, S. Q.; Zhao, Y. L.; Rui, Q.; Wang, D. Y. Toxicity and translocation of graphene oxide in Arabidopsis plants under stress conditions. RSC Adv. 2014, 4, 60891-60901.

[132] Zhao, S. Q.; Wang, Q. Q.; Zhao, Y. L.; Rui, Q.; Wang, D. Y. Toxicity and translocation of graphene oxide in Arabidopsis thaliana. Environ. Toxicol. Pharmacol. 2015, 39, 145-156.

[133] Chen, L. Y.; Wang, C. L.; Li, H. L.; Qu, X. L.; Yang, S. T.; Chang, X. L. Bioaccumulation and toxicity of ${ }^{13} \mathrm{C}$-skeleton labeled graphene oxide in wheat. Environ. Sci. Technol. 2017, 51, 10146-10153.

[134] Zhang, J. Y.; Liu, L. L.; Ren, L.; Feng, W. M.; Lv, P.; Wu, W.; Yan, Y. C. The single and joint toxicity effects of chlorpyrifos and beta-cypermethrin in zebrafish (Danio rerio) early life stages. J. Hazard. Mater. 2017, 334, $121-131$.

[135] Lopes, S.; Pinheiro, C.; Soares, A. M. V. M.; Loureiro, S. Joint toxicity prediction of nanoparticles and ionic counterparts: Simulating toxicity under a fate scenario. J. Hazard. Mater. 2016, 320, 1-9.

[136] Hu, W. B.; Peng, C.; Luo, W. J.; Lv, M.; Li, X. M.; Li, D.; Huang, Q.; Fan, C. H. Graphene-based antibacterial paper. ACS Nano 2010, 4, 4317-4323.

[137] Akhavan, O.; Ghaderi, E. Toxicity of graphene and graphene oxide nanowalls against bacteria. ACS Nano 2010, 4, 5731-5736.

[138] Liu, S. B.; Zeng, T. H.; Hofmann, M.; Burcombe, E.; Wei, J.; Jiang, R. R.; Kong, J.; Chen, Y. Antibacterial activity of graphite, graphite oxide, graphene oxide, and reduced graphene oxide: Membrane and oxidative stress. ACS Nano 2011, 5, 6971-6980.

[139] He, J. L.; Zhu, X. D.; Qi, Z. N.; Wang, C.; Mao, X. J.; Zhu, C. L.; He, Z. Y.; Li, M. Y.; Tang, Z. S. Killing dental pathogens using antibacterial graphene oxide. ACS Appl. Mater. Interfaces 2015, 7, 5605-5611.

[140] Liu, S. B.; Hu, M.; Zeng, T. H.; Wu, R.; Jiang, R. R.; Wei, J.; Wang, L.; Kong, J.; Chen, Y. Lateral dimension-dependent antibacterial activity of graphene oxide sheets. Langmuir 2012, 28, 12364-12372.

[141] Tu, Y. S.; Lv, M.; Xiu, P.; Huynh, T.; Zhang, M.; Castelli, M.; Liu, Z. R.; Huang, Q.; Fan, C. H.; Fang, H. P. et al. Destructive extraction of phospholipids from Escherichia coli membranes by graphene nanosheets. Nat. Nanotechnol. 2013, 8, 594-601.

[142] Perreault, F.; de Faria, A. F.; Nejati, S.; Elimelech, M. Antimicrobial properties of graphene oxide nanosheets: Why size matters. ACS Nano 2015, 9, 7226-7236.

[143] Hui, L. W.; Huang, J. L.; Chen, G. X.; Zhu, Y. W.; Yang, L. H. Antibacterial property of graphene quantum dots (both source material and bacterial shape matter). ACS Appl. Mater. Interfaces 2016, 8, 20-25.

[144] Kuo, W. S.; Chen, H. H.; Chen, S. Y.; Chang, C. Y.; Chen, P. C.; Hou, Y. I.; Shao, Y. T.; Kao, H. F.; Lilian Hsu, C. L.; Chen, Y. C. et al. Graphene quantum dots with nitrogen-doped content dependence for highly efficient dual-modality photodynamic antimicrobial therapy and bioimaging. Biomaterials 2017, 120, 185-194.

[145] Feng, Q. L.; Wu, J.; Chen, G. Q.; Cui, F. Z.; Kim, T. N.; Kim, J. O. A mechanistic study of the antibacterial effect of silver ions on Escherichia coli and Staphylococcus aureus. J. Biomed. Mater. Res. 2000, 52, 662-668.

[146] Das, M. R.; Sarma, R. K.; Borah, S. C.; Kumari, R.; Saikia, R.; Deshmukh, A. B.; Shelke, M. V.; Sengupta, P.; Szunerits, S.; Boukherroub, R. The synthesis of citrate-modified silver nanoparticles in an aqueous suspension of graphene oxide nanosheets and their antibacterial activity. Colloid. Surf. B: Biointerfaces 2013, 105, 128-136.

[147] Yu, L.; Zhang, Y. T.; Zhang, B.; Liu, J. D. Enhanced antibacterial activity of silver nanoparticles/halloysite nanotubes/graphene nanocomposites with sandwich-like structure. Sci. Rep. 2014, 4, 4551.

[148] Shen, J. F.; Shi, M.; Li, N.; Yan, B.; Ma, H. W.; Hu, Y. Z.; Ye, M. X. Facile synthesis and application of Ag-chemically converted graphene nanocomposite. Nano Res. 2010, 3, 339-349.

[149] Prasad, K.; Lekshmi, G. S.; Ostrikov, K.; Lussini, V.; Blinco, J.; Mohandas, M.; Vasilev, K.; Bottle, S.; Bazaka, K.; Ostrikov, K. Synergic bactericidal effects of reduced graphene oxide and silver nanoparticles against Gram-positive and Gram-negative bacteria. Sci. Rep. 2017, 7, 1591.

[150] Xu, W. P.; Zhang, L. C.; Li, J. P.; Lu, Y.; Li, H. H.; Ma, Y. N.; Wang, W. D.; Yu, S. H. Facile synthesis of silver@graphene oxide nanocomposites and their enhanced antibacterial properties. J. Mater. Chem. 2011, 21, 4593-4597.

[151] Ocsoy, I.; Paret, M. L.; Ocsoy, M. A.; Kunwar, S.; Chen, T.; You, M. X.; Tan, W. H. Nanotechnology in plant disease management: DNA-directed silver nanoparticles on graphene oxide as an antibacterial against Xanthomonas perforans. ACS Nano 2013, 7, 8972-8980.

[152] Ocsoy, I.; Gulbakan, B.; Chen, T.; Zhu, G. Z.; Chen, Z.; Sari, M. M.; Peng, L.; Xiong, X. L.; Fang, X. H.; Tan, W. H. DNA-guided metalnanoparticle formation on graphene oxide surface. Adv. Mater. 2013, 25, 2319-2325.

[153] Meng, X. Y.; Wang, H. Y.; Chen, N.; Ding, P.; Shi, H. Y.; Zhai, X.; Su, Y. Y.; He, Y. A graphene-silver nanoparticle-silicon sandwich SERS chip for quantitative detection of molecules and capture, discrimination, and inactivation of bacteria. Anal. Chem. 2018, 90, 5646-5653. 
[154] Wei, Y. H.; Chen, S.; Kowalczyk, B.; Huda, S.; Gray, T. P.; Grzybowski, B. A. Synthesis of stable, low-dispersity copper nanoparticles and nanorods and their antifungal and catalytic properties. J. Phys. Chem. C 2010, 114, $15612-15616$.

[155] Lin, D. H.; Qin, T. Q.; Wang, Y. Q.; Sun, X. Y.; Chen, L. X. Graphene oxide wrapped SERS tags: Multifunctional platforms toward optical labeling, photothermal ablation of bacteria, and the monitoring of killing effect. ACS Appl. Mater. Interfaces 2014, 6, 1320-1329.

[156] Wang, X. D.; Zhou, N. L.; Yuan, J.; Wang, W. Y.; Tang, Y. D.; Lu, C. Y.; Zhang, J.; Shen, J. Antibacterial and anticoagulation properties of carboxylated graphene oxide-lanthanum complexes. J. Mater. Chem. 2012, 22, 1673-1678.

[157] Akhavan, O.; Ghaderi, E. Photocatalytic reduction of graphene oxide nanosheets on $\mathrm{TiO}_{2}$ thin film for photoinactivation of bacteria in solar light irradiation. J. Phys. Chem. C 2009, 113, 20214-20220.

[158] Liu, J. C.; Liu, L.; Bai, H. W.; Wang, Y. J.; Sun, D. D. Gram-scale production of graphene oxide- $\mathrm{TiO}_{2}$ nanorod composites: Towards highactivity photocatalytic materials. Appl. Catal. B: Environ. 2011, 106, $76-82$.

[159] Kim, I. Y.; Park, S.; Kim, H.; Park, S.; Ruoff, R. S.; Hwang, S. J. Strongly-coupled freestanding hybrid films of graphene and layered titanate nanosheets: An effective way to tailor the physicochemical and antibacterial properties of graphene film. Adv. Funct. Mater. 2014, 24, 2288-2294.

[160] Santos, C. M.; Tria, M. C. R.; Vergara, R. A. M. V.; Ahmed, F.; Advincula, R. C.; Rodrigues, D. F. Antimicrobial graphene polymer (PVK-GO) nanocomposite films. Chem. Commun. 2011, 47, 8892-8894.

[161] Some, S.; Ho, S. M.; Dua, P.; Hwang, E.; Shin, Y. H.; Yoo, H.; Kang, J. S.; Lee, D. K.; Lee, H. Dual functions of highly potent graphene derivative-poly-L-lysine composites to inhibit bacteria and support human cells. ACS Nano 2012, 6, 7151-7161.

[162] Li, Y. F.; Yuan, H. Y.; von dem Bussche, A.; Creighton, M.; Hurt, R. H.; Kane, A. B.; Gao, H. J. Graphene microsheets enter cells through spontaneous membrane penetration at edge asperities and corner sites. Proc. Natl. Acad. Sci. USA 2013, 110, 12295-12300.

[163] Pham, V. T. H.; Truong, V. K.; Quinn, M. D. J.; Notley, S. M.; Guo, Y. C.; Baulin, V. A.; Al Kobaisi, M.; Crawford, R. J.; Ivanova, E. P. Graphene induces formation of pores that kill spherical and rod-shaped bacteria. ACS Nano 2015, 9, 8458-8467.

[164] Yi, X.; Gao, H. J. Cell interaction with graphene microsheets: Nearorthogonal cutting versus parallel attachment. Nanoscale 2015, 7, 5457-5467.

[165] Dallavalle, M.; Calvaresi, M.; Bottoni, A.; Melle-Franco, M.; Zerbetto, F. Graphene can wreak havoc with cell membranes. ACS Appl. Mater. Interfaces 2015, 7, 4406-4414.

[166] Mangadlao, J. D.; Santos, C. M.; Felipe, M. J. L.; de Leon, A. C. C.; Rodrigues, D. F.; Advincula, R. C. On the antibacterial mechanism of graphene oxide (GO) Langmuir-Blodgett films. Chem. Commun. 2015, $51,2886-2889$.

[167] Hui, L. W.; Piao, J. G.; Auletta, J.; Hu, K.; Zhu, Y. W.; Meyer, T.; Liu, H. T.; Yang, L. H. Availability of the basal planes of graphene oxide determines whether it is antibacterial. ACS Appl. Mater. Interfaces 2014, 6, 13183-13190.

[168] Tan, K. H.; Sattari, S.; Donskyi, I. S.; Cuellar-Camacho, J. L.; Cheng, C.; Schwibbert, K.; Lippitz, A.; Unger, W. E. S.; Gorbushina, A.; Adeli, M. et al. Functionalized 2D nanomaterials with switchable binding to investigate graphene-bacteria interactions. Nanoscale 2018, 10, 9525-9537.

[169] Lyon, D. Y.; Brunet, L.; Hinkal, G. W.; Wiesner, M. R.; Alvarez, P. J. J. Antibacterial activity of fullerene water suspensions $\left(\mathrm{nC}_{60}\right)$ is not due to ROS-mediated damage. Nano Lett. 2008, 8, 1539-1543.

[170] Niu, A. P.; Han, Y. J.; Wu, J.; Yu, N.; Xu, Q. Synthesis of onedimensional carbon nanomaterials wrapped by silver nanoparticles and their antibacterial behavior. J. Phys. Chem. C 2010, 114, 12728-12735.

[171] West, J. D.; Marnett, L. J. Endogenous reactive intermediates as modulators of cell signaling and cell death. Chem. Res. Toxicol. 2006, 19, 173-194.

[172] Gurunathan, S.; Han, J. W.; Dayem, A. A.; Eppakayala, V.; Kim, J. H. Oxidative stress-mediated antibacterial activity of graphene oxide and reduced graphene oxide in Pseudomonas aeruginosa. Int. J. Nanomedicine 2012, 7, 5901-5914.
[173] Pang, W. C.; Wu, J. L.; Zhang, Q. F.; Li, G. F. Graphene oxide enhanced, radiation cross-linked, vitamin $\mathrm{E}$ stabilized oxidation resistant UHMWPE with high hardness and tensile properties. RSC Adv. 2017, 7, 55536-55546.

[174] Chen, J. N.; Wang, X. P.; Han, H. Y. A new function of graphene oxide emerges: Inactivating phytopathogenic bacterium Xanthomonas oryzae pv. Oryzae. J. Nanopart. Res. 2013, 15, 1658.

[175] Gurunathan, S.; Han, J. W.; Dayem, A. A.; Eppakayala, V.; Park, M. R.; Kwon, D. N.; Kim, J. H. Antibacterial activity of dithiothreitol reduced graphene oxide. J. Ind. Eng. Chem. 2013, 19, 1280-1288.

[176] Krishnamoorthy, K.; Veerapandian, M.; Zhang, L. H.; Yun, K.; Kim, S. J. Antibacterial efficiency of graphene nanosheets against pathogenic bacteria via lipid peroxidation. J. Phys. Chem. C 2012, 116, 17280-17287.

[177] Giglio, S.; Jiang, J. Y.; Saint, C. P.; Cane, D. E.; Monis, P. T. Isolation and characterization of the gene associated with geosmin production in cyanobacteria. Environ. Sci. Technol. 2008, 42, 8027-8032.

[178] Li, J. H.; Wang, G.; Zhu, H. Q.; Zhang, M.; Zheng, X. H.; Di, Z. F.; Liu, X. Y.; Wang, X. Antibacterial activity of large-area monolayer graphene film manipulated by charge transfer. Sci. Rep. 2014, 4, 4359.

[179] Carpio, I. E. M.; Santos, C. M.; Wei, X.; Rodrigues, D. F. Toxicity of a polymer-graphene oxide composite against bacterial planktonic cells, biofilms, and mammalian cells. Nanoscale 2012, 4, 4746-4756.

[180] Chen, J. N.; Peng, H.; Wang, X.P.; Shao, F.; Yuan, Z. D.; Han, H. Y. Graphene oxide exhibits broad-spectrum antimicrobial activity against bacterial phytopathogens and fungal conidia by intertwining and membrane perturbation. Nanoscale 2014, 6, 1879-1889.

[181] Kanchanapally, R.; Nellore, B. P. V.; Sinha, S. S.; Pedraza, F.; Jones, S. J.; Pramanik, A.; Chavva, S. R.; Tchounwou, C.; Shi, Y. L.; Vangara, A. et al. Antimicrobial peptide-conjugated graphene oxide membrane for efficient removal and effective killing of multiple drug resistant bacteria. RSC Adv. 2015, 5, 18881-18887.

[182] Akhavan, O.; Ghaderi, E.; Esfandiar, A. Wrapping bacteria by graphene nanosheets for isolation from environment, reactivation by sonication, and inactivation by near-infrared irradiation. J. Phys. Chem. B 2011, 115, 6279-6288.

[183] Luan, B. Q.; Huynh, T.; Zhao, L.; Zhou, R. H. Potential toxicity of graphene to cell functions via disrupting protein-protein interactions. ACS Nano 2015, 9, 663-669.

[184] Zhao, J. M.; Deng, B.; Lv, M.; Li, J. Y.; Zhang, Y. J.; Jiang, H. Q.; Peng, C.; Li, J.; Shi, J. Y.; Huang, Q. et al. Graphene oxide-based antibacterial cotton fabrics. Adv. Healthc. Mater. 2013, 2, 1259-1266.

[185] Karimi, L.; Yazdanshenas, M. E.; Khajavi, R.; Rashidi, A.; Mirjalili, M. Using graphene $/ \mathrm{TiO}_{2}$ nanocomposite as a new route for preparation of electroconductive, self-cleaning, antibacterial and antifungal cotton fabric without toxicity. Cellulose 2014, 21, 3813-3827.

[186] Fan, Z. J.; Liu, B.; Wang, J. Q.; Zhang, S. Y.; Lin, Q. Q.; Gong, P. W.; Ma, L. M.; Yang, S. R. A novel wound dressing based on Ag/graphene Adv. Funct. Mater. 2014, 24, 3933-3943.

[187] Zhou, Y. Z.; Chen, R.; He, T. T.; Xu, K.; Du, D.; Zhao, N.; Cheng, X. N.; Yang, J.; Shi, H. F.; Lin, Y. H. Biomedical potential of ultrafine $\mathrm{Ag} / \mathrm{AgCl}$ nanoparticles coated on graphene with special reference to antimicrobial performances and burn wound healing. ACS Appl. Mater. Interfaces 2016, 8, 15067-15075.

[188] Lu, B. G.; Li, T.; Zhao, H. T.; Li, X. D.; Gao, C. T.; Zhang, S. X.; Xie, E. Q. Graphene-based composite materials beneficial to wound healing. Nanoscale 2012, 4, 2978-2982.

[189] Sun, X. M.; Liu, Z.; Welsher, K.; Robinson, J. T.; Goodwin, A.; Zaric, S.; Dai, H. J. Nano-graphene oxide for cellular imaging and drug delivery. Nano Res. 2008, 1, 203-212.

[190] Pandey, H.; Parashar, V.; Parashar, R.; Prakash, R.; Ramteke, P. W.; Pandey, A. C. Controlled drug release characteristics and enhanced antibacterial effect of graphene nanosheets containing gentamicin sulfate. Nanoscale 2011, 3, 4104-4108.

[191] Wang, Y.; Zhang, D.; Bao, Q.; Wu, J. J.; Wan, Y. Controlled drug release characteristics and enhanced antibacterial effect of graphene oxide-drug intercalated layered double hydroxide hybrid films. J. Mater. Chem. 2012, 22, 23106-23113.

[192] Ghadim, E. E.; Manouchehri, F.; Soleimani, G.; Hosseini, H.; Kimiagar, S.; Nafisi, S. Adsorption properties of tetracycline onto graphene oxide: Equilibrium, kinetic and thermodynamic studies. PLoS One 2013, 8, e79254. 
[193] Huang, T. F.; Zhang, L.; Chen, H. L.; Gao, C. J. A cross-linking graphene oxide-polyethyleneimine hybrid film containing ciprofloxacin: One-step preparation, controlled drug release and antibacterial performance. $J$. Mater. Chem. B 2015, 3, 1605-1611.

[194] Ding, H.; Zhang, F.; Zhao, C. C.; Lv, Y. L.; Ma, G. H.; Wei, W.; Tian, Z. Y. Beyond a carrier: Graphene quantum dots as a probe for programmatically monitoring anti-cancer drug delivery, release, and response. ACS Appl. Mater. Interfaces 2017, 9, 27396-27401.

[195] Mo, R.; Jiang, T.; Sun, W.; Gu, Z. ATP-responsive DNA-graphene hybrid nanoaggregates for anticancer drug delivery. Biomaterials 2015, 50, $67-74$.

[196] Jiang, T.; Sun, W.; Zhu, Q.; Burns, N. A.; Khan, S. A.; Mo, R.; Gu, Z. Furin-mediated sequential delivery of anticancer cytokine and smallmolecule drug shuttled by grapheme. Adv. Mater. 2015, 27, 1021-1028.

[197] Zeng, X. K.; McCarthy, D. T.; Deletic, A.; Zhang, X. W. Silver/reduced graphene oxide hydrogel as novel bactericidal filter for point-of-use water disinfection. Adv. Funct. Mater. 2015, 25, 4344-4351.

[198] Zeng, X. K.; Wang, Z. Y.; Meng, N.; McCarthy, D. T.; Deletic, A.; Pan, J. H.; Zhang, X. W. Highly dispersed $\mathrm{TiO}_{2}$ nanocrystals and carbon dots on reduced graphene oxide: Ternary nanocomposites for accelerated photocatalytic water disinfection. Appl. Catal. B: Environ. 2017, 202, $33-41$.
[199] Zeng, X. K.; Wang, Z. Y.; Wang, G.; Gengenbach, T. R.; McCarthy, D. T.; Deletic, A.; Yu, J. G.; Zhang, X. W. Highly dispersed $\mathrm{TiO}_{2}$ nanocrystals and $\mathrm{WO}_{3}$ Nanorods on reduced graphene oxide: $\mathrm{Z}$-scheme photocatalysis system for accelerated photocatalytic water disinfection. Appl. Catal. B: Environ. 2017, 218, 163-173.

[200] Nellore, B. P. V.; Kanchanapally, R.; Pedraza, F.; Sinha, S. S.; Pramanik, A.; Hamme, A. T.; Arslan, Z.; Sardar, D.; Ray, P. C. Bio-conjugated CNT-bridged 3D porous graphene oxide membrane for highly efficient disinfection of pathogenic bacteria and removal of toxic metals from water. ACS Appl. Mater. Interfaces 2015, 7, 19210-19218.

[201] Armani, M. A.; Abu-Taleb, A.; Remalli, N.; Abdullah, M.; Srikanth, V. V. S. S.; Labhasetwar, N. K. Dragon's blood-aided synthesis of $\mathrm{Ag} / \mathrm{Ag}_{2} \mathrm{O}$ core/shell nanostructures and $\mathrm{Ag} / \mathrm{Ag}_{2} \mathrm{O}$ decked multi-layered graphene for efficient As(III) uptake from water and antibacterial activity. RSC Adv. 2016, 6, 44145-44153.

[202] Wang, Y. L.; El-Deen, A. G.; Li, P.; Oh, B. H. L.; Guo, Z. R.; Khin, M. M.; Vikhe, Y. S.; Wang, J.; Hu, R. G.; Boom, R. M. et al. High-performance capacitive deionization disinfection of water with graphene oxidegraft-quaternized chitosan nanohybrid electrode coating. ACS Nano 2015, 9, 10142-10157. 